\title{
Identificación geográfica de barrios residenciales a partir de la actividad extractiva del carbón en Río Gallegos desde el siglo XX
}

\section{Geographical identification of residential neighborhoods from the extractive activity of coal in Rio Gallegos since the 20th century}

\author{
Karla Lizeth Aceves Padilla, Klap80@ hotmail.com \\ Alicia Pompeya Cáceres, apcaceres@gmail.com
}

\author{
Universidad Nacional de la Patagonia Austral. Unidad Académica Río Gallegos \\ Río Gallegos, Santa Cruz, Argentina
}

Recibido: 23/03/2017. Aceptado: 20/03/2018

\begin{abstract}
RESUMEN
El crecimiento de la ciudad de Río Gallegos ha estado vinculado a las actividades económicas que se han desarrollado en el paso del tiempo. Actualmente los barrios delimitados por la municipalidad, no corresponden con la identidad que se ha desarrollado en un área determinada. Por esta razón fue necesario identificar los barrios surgidos a partir de la actividad extractiva del carbón, analizar si corresponde con la delimitación municipal del Barrio El Trencito y detectar aquellos sectores que no hayan sido tomados en cuenta. Se identificaron manzanas que contenían viviendas cuyo origen está relacionado con la empresa Yacimientos Carboníferos Fiscales, misma que se encarga de toda la dinámica del carbón. Al tratarse de una extensa área, estas manzanas debían cumplir tres criterios: tener un vínculo con YCF, haber sido nombradas por los testimonios recopilados y contar con un respaldo legal. Se reconoce su construcción en cuatro periodos de tiempo diferente, y se concentran no solo en el Barrio El Trencito. Se trata de una investigación archivística e histórica, basada en búsqueda bibliográfica, de documentos oficiales y de testimonios que ayudaron a reconstruir los hechos y georeferenciarlos a través de un SIG. Se contó con planos de mensura, y software de SIG, para la realización.

El trabajo consta de un marco de referencia con información de Río Gallegos, Río Turbio e YCF. Posteriormente se expone la metodología, los resultados y análisis, donde se incluye una cartografía con la identificación de las manzanas y su periodo de construcción, y por último las conclusiones y recomendaciones.
\end{abstract}

Palabras clave: Barrios; Carbón; YCF; YCRT; Río Gallegos.

\begin{abstract}
The growth of the city of Río Gallegos has been related to the economic activities that have developed over time. Currently the neighborhoods delimited by the municipality, does not correspond with the identity that has developed in a determined area. For this reason it was necessary to identify the neighborhoods arising from the extractive activity of the coal, to analyze if it corresponds with the municipal delimitation of the Neighborhood El Trencito and to detect those sectors that have not been taken into account. They identified blocks containing housing whose origin is related to the company Yacimientos Carboníferos Fiscales, which is responsible for all the dynamics of coal. As a large area, these blocks had to
\end{abstract}


fulfill three conditions: to be related to YCF, to have been named by the testimonies collected and to have legal support. Its construction is recognized in four different periods of time, and are concentrated not only in the El Trencito neighborhood. It is an archival and historical investigation, based on a bibliographical search, official documents and testimonies that helped to reconstruct the facts and georeferenced them through a GIS. Plans of measurement, and GIS software, were counted for the realization.

The work consists of a frame of reference with information from Río Gallegos, Río Turbio and YCF. Subsequently, the methodology, results and analysis are presented, which includes a mapping with the identification of the blocks and their construction period, and finally the conclusions and recommendations.

Key words: Neighborhood; Coal; YCF; YCRT; Río Gallegos.

\section{INTRODUCCIÓN}

La presente investigación se enmarca dentro del Proyecto de Investigación PI 29/314 "Delimitación de barrios residenciales a partir de criterios geográficos, históricos y urbanísticos en Río Gallegos, ciudad intermedia de la Patagonia Austral”, en la Unidad Académica Río Gallegos, de la Universidad Nacional de la Patagonia Austral, Argentina.

El crecimiento de la ciudad de Río Gallegos ha estado vinculado a las actividades económicas que se han desarrollado en el paso del tiempo, actividades que van dejando huella y que van definiendo la identidad de la ciudad y de sus sectores internos. Actualmente estos sectores internos, están delimitados en barrios por la municipalidad, delimitación que muchas veces no corresponde con la identidad que se ha desarrollado a través del tiempo en un área determinada, dejando fuera sectores que, por sus orígenes y su historia, deberían estar comprendidos en una delimitación de barrio determinada. Por esta razón fue necesaria la realización de esta investigación para reconocer aquellos barrios o manzanas surgidos a partir de la actividad extractiva del carbón, analizar si corresponde con la delimitación municipal del Barrio El Trencito y detectar aquellos sectores que no hayan sido tomados en cuenta. Se identificaron manzanas que contenían viviendas cuyo origen está relacionado con la empresa Yacimientos Carboníferos Fiscales, misma que se encarga de toda la dinámica del carbón. Al tratarse de una extensa área, estas manzanas fueron elegidas con tres criterios: debían tener un vínculo con YCF, haber sido nombradas por los testimonios recopilados y contar con un respaldo legal. Se edificaron en cuatro periodos de tiempos diferente, y concentradas no solo en el Barrio El Trencito. Queda para futuras investigaciones la realización de una propuesta de delimitación dada la distancia física existente entre las manzanas. Se trata de una investigación basada en búsqueda bibliográfica, de documentos oficiales y de testimonios que ayudaron a reconstruir históricamente los hechos y georeferenciarlos en un SIG. Se contó con computadora, planos de mensura, diferentes software, y SIG, entre otros, para la realización. Este informe puede ser considerado como una segunda parte del trabajo "Identificación geográfica de barrios residenciales surgidos a partir de la actividad hidrocarburífera en Río Gallegos durante el siglo XX, realizado por Aceves y Cáceres en una beca UNPA en el año 2015.

Dados los criterios establecidos para la elección de las manzanas, pudieron haber quedado muchas otras fuera de este estudio, por lo que se sugiere hacer una segunda investigación sobre este tema. Así mismo, se pide que los resultados sean tratados con toda la responsabilidad profesional necesaria, esto implica, no alterarlos, ni reproducirlos sin citar a las autoras. 
El trabajo consta de un marco de referencia donde se encuentra información históricageográfica de Río Gallegos y Río Turbio, sin dejar de lado la empresa Yacimientos Carboníferos Fiscales. Posteriormente se exponen los resultados y análisis con su correspondiente metodología, y por último las conclusiones y recomendaciones.

\section{MARCOS DE REFERENCIA}

\subsection{Marco histórico - geográfico}

La presente investigación se enmarca dentro del Programa de Becas de Iniciación a la Investigación para estudiantes de grado y pregrado, vinculado al PI 29/314 "Delimitación de barrios residenciales a partir de criterios geográficos, históricos y urbanísticos en Río Gallegos, ciudad intermedia de la Patagonia Austral", del Instituto de Cs. Del Ambiente, Sustentabilidad y Recursos Naturales (ICASUR).

Como antecedentes del tema de investigación se encuentran: con relación a barrios surgidos de actividades extractivas, el informe científico técnico que lleva por título "Identificación geográfica de barrios residenciales surgidos a partir de la actividad hidrocarburífera en Río Gallegos durante el siglo XX" del año 2015, de Aceves y Cáceres; el trabajo sobre las casas de durmientes de YCF en Río Gallegos de César Ferro del año 2006; la delimitación de los barrios construidos por el Estado Nacional, Provincial y Municipal de Andrea Menco. Así como también varios estudios realizados en el norte de Santa Cruz y en Comodoro Rivadavia sobre áreas pobladas como consecuencia de la explotación del petróleo, entre otros.

Las áreas analizadas en este informe se encuentran en la planta urbana perteneciente al ejido municipal de la ciudad de Rio Gallegos en el Departamento de Güer Aike, Provincia de Santa Cruz, Argentina. Este informe científico técnico se compone del marco de referencia en el cual se incluye una breve descripción geográfica e histórica de Río Gallegos y Río Turbio, destacando la función que ha tenido la empresa estatal Yacimientos Carboníferos Fiscales/Yacimientos Carboníferos Río Turbio (en adelante YCF o YCRT respectivamente). Posteriormente se presentarán los resultados, el análisis y las conclusiones.

\section{Ubicación geográfica de Río Gallegos}

La ciudad de Río Gallegos es la capital de la provincia de Santa Cruz, en el Sur de Argentina. Sus coordenadas geográficas son $51^{\circ} 37^{\prime} 27^{\prime}$ ' Latitud sur y $69^{\circ} 12^{\prime} 59^{\prime}$ ' Longitud oeste (figura 1). De acuerdo al INDEC, el censo del año 2010 arrojó que el Departamento de Güer Aike, al cual pertenece Río Gallegos, tenía 113267 habitantes, y la estimación para el año 2016 es de 129110 habitantes.

Su emplazamiento está en la margen derecha del estuario de Río Gallegos sobre terrazas fluviales formadas por sedimentos fluviales y fluvioglaciales, con pendiente de sudoeste a noreste. La altura media está por debajo de los 20 m.s.n.m. De acuerdo a la clasificación de Cabrera Willink, pertenece al distrito Patagonia Subandina, predominando la estepa magallánica, con ausencia absoluta de árboles, característica del clima frío semiárido de meseta. Se dan vientos dominantes del sector oeste-sudoeste, siendo su velocidad promedio de $35 \mathrm{~km} / \mathrm{h}$ en enero y $18 \mathrm{~km} / \mathrm{h}$ en junio. Las precipitaciones a lo largo del año oscilan entre los 200 y $300 \mathrm{~mm}$. La presencia de vientos fuertes se explica por encontrarse en una zona de libre circulación atmosférica y oceánica. Por su posición latitudinal los días son largos en el verano y cortos en el invierno. Se caracteriza, por la aridez, los vientos fuertes y las mesetas de suave relieve, generando un paisaje monótono y hostil, siendo un condicionante de vida. Al 
estar situada al sureste de la provincia de Santa Cruz y del país, su posición es austral, extrema y periférica. (Cáceres et al., 1996).

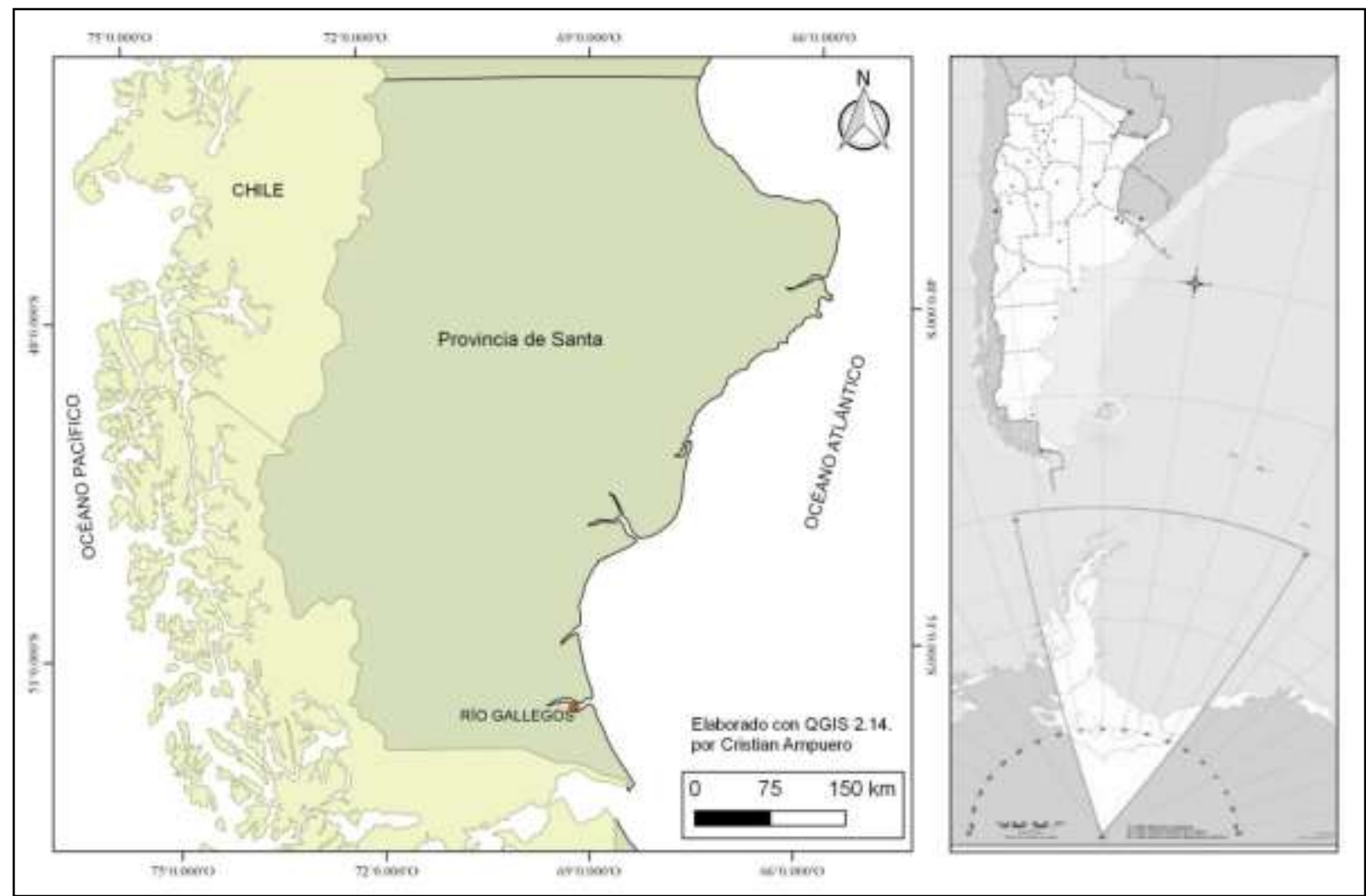

Figura 1. Ubicación geográfica de Río Gallegos y Río Turbio. Elaborado por Cristian Ampuero.

Al ser capital provincial es sede de los poderes Ejecutivo, Legislativo y Judicial, destacándose por su función administrativa. Por la presencia de numerosos comercios, centros universitarios, colegios, medios de comunicación, asociaciones de distinta índole, y por tener infraestructura para actividades recreativas, deportivas y sociales se ha convertido en un centro comercial y de influencia en el extremo sur de la Patagonia (Aguirre, S. 2000).

La comunicación de la ciudad con el resto de la provincia y del país es facilitada por tres vías: terrestre, aérea y marítima. Vía terrestre: la comunicación hacia el país y a Chile es favorecida por dos importantes rutas nacionales, la ruta nacional $\mathrm{N}^{\circ} 40$ que comunica al oeste con las localidades de la Cuenca Carbonífera Río Turbio y 28 de Noviembre en territorio argentino y Puerto Natales en Chile; y la ruta nacional $N^{\circ} 3$ que permite la comunicación hacia el norte con el resto del país, hacia el sur con la provincia de Tierra del Fuego y hacia el Sudoeste con la ciudad de Punta Arenas, Chile. A estas importantes rutas se agrega, una ruta provincial destacada, la ruta provincial $\mathrm{N}^{\circ} 5$ que comunica con los centros turísticos provinciales de $\mathrm{El}$ Calafate, -al Glaciar Perito Moreno- y de El Chaltén. Por la confluencia de estas vías de circulación define su situación de encrucijada (Cáceres y García, 2004). Vía aérea: la mayoría de los vuelos conectan a la ciudad con Buenos Aires, la capital del país, además de Comodoro Rivadavia, Ushuaia, las Islas Malvinas y la ciudad de Punta Arenas en Chile. Por vía marítima, se cuenta con el puerto de Punta Loyola, que dista 50 kilómetros de la ciudad, y es a donde los barcos llegan para el transporte del carbón proveniente de la localidad de Río Turbio por vía férrea, conformando, así, un ramal ferro industrial. Tanto el transporte marítimo como el férreo no son usados para el transporte de pasajeros (Aguirre, S. 2000). 


\section{Historia de Río Gallegos}

Río Gallegos comenzó formalmente a fines del siglo XIX con el establecimiento de una Subprefectura Marítima el 19 de diciembre de 1885. El poblamiento se fue dando con la ocupación de tierras que se entregaron al sur del Río Gallegos para la ganadería ovina, cuya producción de lana se exportaba a Europa. Un factor importante que influyó para hacer de Río Gallegos la capital fue el descubrimiento de oro en la zona de Cabo Vírgenes. Para el año de 1895, en el Departamento de Río Gallegos había 25 casas y la población era de 145 habitantes. La gente venida de Punta Arenas y de Islas Malvinas eran la mayoría de los pobladores. Se dan las primeras viviendas particulares y oficiales, que se traían de Punta Arenas, así lo refiere Alicia N. García (2000). Las primeras manzanas dispuesta en cuadrícula, con la plaza central, en cuyo alrededor se ubicaron los edificios públicos más importantes denotan la influencia de la época colonial (tardía). (Perazzo, 1985). La actividad en el área urbana se relacionaba con la función del gobierno o con el incipiente comercio.

Según García (2000), en los primeros años se dan los primeros pasos para la organización de la enseñanza pública y la policía territorial. Se crea el Juzgado Letrado, el Registro Civil, un hospital, los primeros clubes; bancos, iglesia, comercios que son la base de la población urbana estable, hoteles. Desde 1895, se acelera el proceso de crecimiento en el ámbito urbano, que se observa en el aumento de la población, en una progresiva ocupación de lotes urbanos ${ }^{1}$ y en el incremento de la actividad comercial. En 1899, la gobernación ordenó construir un muelle al que se denominó muelle fiscal, y por su parte la Sociedad Anónima construyó otro en 1913 denominado más tarde Enosis. Al finalizar el siglo se realiza el ordenamiento catastral que servirá de base al crecimiento de la ciudad durante varias décadas. En cuanto al trazado de la planta urbana, el Ing. Williams decidió que el ancho de las calles fuera de 25 m., respetando las ya existentes. Las manzanas fueron divididas en cuatro solares a, b, c, d. (esta traza aún se puede ver en los registros catastrales). Una característica respecto a la acumulación de tierras en el ámbito urbano es la ubicación de las mismas, ya que los concesionarios trataron de obtener sus posesiones combinando la ubicación en la zona del puerto y en la zona comercial que comenzaba a formarse sobre la Avenida Roca. Hasta 1912 la vida institucional de la localidad estaba regida por las autoridades territoriales, de acuerdo con los dispuesto en la Ley 1532. En 1912 se elige un Concejo Municipal, que continúa en funcionamiento hasta la provincialización.

A fines de la década del 40, se destina a la Secretaría de Aeronáutica 330 ha., para la construcción de la Base Aérea Militar Patagónica Sur y en 1948 la Dirección General de Infraestructura Aeronáutica encara la construcción de casas para oficiales, suboficiales y obreros que constituirán la dotación de la futura Base Aérea. También se destinan varias manzanas de la ciudad para la construcción de viviendas del personal de la Prefectura General Marítima, para el personal de la cárcel y se cede a YPF la manzana 267 para instalar en ella su campamento, casillas para viviendas de empleados y obreros. El crecimiento de la población fue sostenido, manteniéndose siempre en el primer lugar en cuanto a la cantidad de habitantes. El censo nacional de 1947 arrojó 5880 habitantes y para 1960 había ascendido a 14439 pobladores.

El aumento de la población requería de la habilitación de nuevos servicios en educación y salud. Económicamente se mantenía la importancia de la actividad ganadera y en el núcleo urbano, la actividad del frigorífico constituía una importante fuente de trabajo, pero además el asiento de la administración de YCF y de los campamentos de YPF, atrajeron una nueva oleada de pobladores, provenientes de otras provincias argentinas y de Chile. Uno de los

\footnotetext{
${ }^{1}$ Según Barbería (1985), la ocupación de la tierra en la zona es a través de dos formas de tenencia: propiedad y arrendamiento.
} 
problemas que se debía afrontar por el incremento constante de la población era la escasez de viviendas. Río Gallegos además al ser confirmada como Capital de la Provincia, se mantuvo como sede de los tres Poderes y recibió la afluencia de nuevos pobladores. En esta etapa surgen también distintas entidades cooperativas, como las mutualistas de principios de siglo que buscan mejorar las condiciones de vida. En 1969 se realiza el tendido de la red de gas domiciliaria, con lo que se modifica un aspecto característico de la vida cotidiana de los habitantes y de la ciudad: al despertar, era percibido el humo de las chimeneas y caños de las estufas a carbón. En las décadas siguientes, la población continúa aumentando, por lo que la planta urbana comienza a ser ocupada de modo intensivo. En la década del ochenta uno de los mayores problemas es la falta de terrenos para uso residencial, lo cual obliga a la realización de gestiones para recuperar desde el Municipio, que administra la tierra urbana desde la provincialización, las tierras otorgadas a las Fuerzas Armadas, cuyas instalaciones alrededor de la planta urbana actuaban como una faja que impedía la expansión de la misma. El censo nacional de 1991, registró 64852 habitantes, con crecimiento del 49,2\% en relación al de 1980. En esas últimas dos décadas el aspecto de la localidad ha cambiado, notándose un mayor interés por el embellecimiento de los frentes de las viviendas y un incremento en la forestación y jardines. En la década del 1980 se inaugura el paseo costanero, modificando el aspecto de la zona portuaria en donde estaban ubicadas algunas casas del frigorífico, y el paisaje estaba dominado por el canto rodado.

García describe que a mitad del siglo XX, se asentaron en Río Gallegos las empresas de YPF e YCRT, a partir del impulso que los gobiernos nacionales decidieron dar a los yacimientos petroleros y carboníferos para fortalecer la matriz energética del país. Esta última empresa es la que interesa a los fines de este trabajo de investigación, y hablar de YCF, es hablar también de la localidad de Río Turbio, donde se encuentra el yacimiento de carbón.

\section{Río Turbio}

En la zona cordillerana, vinculados a la explotación del carbón, que se inicia en la década de 1940, surgen nuevas localidades ubicadas en el extremo sur del eje cordillerano, entre ellas Río Turbio, aunque el poblamiento del área se inicia a principios del siglo con la aparición de establecimientos ganaderos (García, 2000).

De acuerdo al trabajo de Muñoz Sesnic, Norambuena y Cáceres (2011), Río Turbio se sitúa a los $51^{\circ} 34^{\prime} 07^{*}$ S y $72^{\circ} 20^{\prime} 51^{*}$ O, en la Patagonia austral andina argentina al suroeste de la provincia de Santa Cruz. La situación geográfica se caracteriza por ser parte de un eje de comunicaciones constituido por la explotación del yacimiento de carbón mineral. Se emplaza en el interfluvio de subafluentes del tramo superior de la cuenca hidrográfica del río Gallegos, sobre un relieve de transición entre la alta meseta patagónica y la cordillera de pendientes suaves que oscilan entre los 250 y $750 \mathrm{msnm}$. Posee un clima frío, húmedo, con vientos intensos del oeste y temperatura media anual de $5.7^{\circ} \mathrm{C}$. La vegetación predominante es el nothofagus. La fauna es de pumas, gatos, zorros, liebres, entre otros. El área urbana ocupa el $80 \%$ del ejido municipal donde los usos de suelo (residencial, comercial y administrativo) están asociados a la extracción del carbón mineral, por tanto las fluctuaciones de la actividad carbonífera inciden directamente en su dinamismo. La presencia de servicios como el educativo hasta el nivel universitario, le permite establecer cada vez más vínculos con el sector social, empresarial, rural y minero. El Estado Nacional y el Provincial aparecen como promotores del desarrollo de la región. Actualmente, la mayor demanda de empleo es de la empresa YCRT.

García (2000) expone una interesante narración sobre la historia de Río Turbio, destacando la vinculación existente entre el desarrollo de la localidad y la empresa YCF. Guillermo Greenwood bautizó como tal a Río Turbio en el año de 1883. En 1887 y 1888 Agustin de 
Castillo realiza viajes a Río Turbio confirmando lo que años atrás Carlos M. Moyano había señalado sobre la presencia de carbón en la zona. Con estas exploraciones queda asentada la existencia del yacimiento que más tarde daría origen a una serie de asentamientos poblados que conforman el complejo minero de la cuenca carbonífera. En 1920 se instala el primer caserío de la zona, originado por la importancia de la actividad ganadera, se establece un Hotel en El Turbio, (hoy Turbio Viejo) y poco después se establece en las cercanías el Juzgado de Paz y la comisaría. Al iniciarse los trabajos relacionados con la explotación del carbón Santa Cruz, mantenía la configuración adquirida durante su proceso de ocupación, estrechamente ligado a la producción ovina. Entre las causas que impulsan la explotación del carbón se encuentra la crisis energética que trajo aparejada la segunda guerra mundial a los países que se abastecían en el mercado europeo lo que indujo al Gobierno Nacional a buscar soluciones que tendieran al autoabastecimiento en materia energética. En consecuencia el Estado dispuso el inicio de trabajos de exploración en aquéllas zonas en que se contaba con recursos que permitieran solucionar la coyuntura. Entre las medidas adoptadas en ese momento fueron las normas legales que se dictaron en relación con la explotación del carbón, entre las que se encuentra el Decreto 87672 de 1941, por el cual se faculta a YPF a realizar los estudios necesarios para determinar las características y explotabilidad de los yacimientos de carbón. Durante los primeros años la explotación del yacimiento se mantuvo en la órbita de YPF hasta 1958 en que se crea la Empresa Estatal YCF por Decreto 3686. Para entonces el Yacimiento de Río Turbio se encontraba en funcionamiento y se había avanzado en obras de infraestructura tanto para el laboreo minero como para el transporte de la producción. En 1959 se firma un convenio entre el Presidente de YCF y el gobernador de la Provincia en el que se transfieren a ésta, parte de las tierras expropiadas y por el cual la Provincia asume el compromiso de asegurar el abastecimiento de carne y otros alimentos para la población. El Estado nacional también tuvo a su cargo el tendido de las vías de ferrocarril, a través del Ministerio de Transporte de la Nación, obras que se llevaron a cabo entre 1950 y 1951. Estas y todas las obras destinadas a mejorar las condiciones de explotabilidad se mantuvieron desde su inicio en la órbita del Estado Nacional. Es importante señalar que al iniciarse los trabajos, la crisis energética imponía la búsqueda de soluciones inmediatas; sin embargo la ubicación periférica del yacimiento y la ausencia total de infraestructura no permitieron que la producción del carbón llegara al mercado nacional sino después de que los factores coyunturales, que habían acelerado la decisión de explotar este recurso, se habían superado.

Aunque la necesidad de autoabastecimiento energético además de la necesidad de reforzar la soberanía en una zona limítrofe, mantuvo el interés del Estado en esta explotación, así lo indica su inclusión en el Plan Quinquenal durante el gobierno peronista, la prosecución de las obras portuarias en Río Gallegos y la creación de YCF en 1958. Como se ha señalado, la explotación del carbón obedeció a la crisis energética suscitada por la segunda guerra; ésta afectaba también el abastecimiento en esta zona, por lo que la producción de carbón en Río Turbio abría una posibilidad para solucionar el abastecimiento del propio territorio, aún cuando la ubicación de la mina no permitiera que el abastecimiento se haga efectivo en forma inmediata. Para dar impulso a la explotación carbonífera fue necesario el mejoramiento de las vías de comunicación.

El traslado de la producción se realizó durante los primeros años por medio de camiones a través de la Ruta 293. La solución definitiva llegaría con el tendido de las vías del ferrocarril, lo que se concretó en 1951. Las primeras extracciones estuvieron destinadas a las estancias de la zona y al abastecimiento de Río Gallegos. En 1958 se inaugura el puerto que permitiría la salida hacia otras zonas. A raíz de la explotación carbonífera se inicia un proceso de crecimiento de la población basado en el personal trasladado por el Estado para los trabajos propios del laboreo minero y por la llegada de inmigrantes. Debido a la cercanía con la localidad chilena de Puerto Natales, muchos de ellos provenían del país limítrofe además de 
los contingentes de italianos que se establecieron en la región, en 1948. Las instalaciones necesarias para el establecimiento de la población fueron realizadas por el Estado, quien tuvo a su cargo la construcción de viviendas para el personal, y todos los servicios relacionados con el funcionamiento de la villa minera.

Además de las obras relacionadas con la producción, la empresa atendía las necesidades del personal en lo que se refiere a viviendas, salud, educación y también esparcimiento. La explotación del carbón trajo aparejado un crecimiento de la población que estuvo íntimamente ligado con el desarrollo de la misma. El personal de la empresa estuvo conformado por un importante número de extranjeros; italianos, bolivianos y en mayor porcentaje, chilenos. Estos últimos, debido a la cercanía de la localidad limítrofe de Puerto Natales y al régimen de propiedad de la tierra en zonas de frontera, se convirtieron en una suerte de "trabajadores golondrina" habitando en las gemelas para solteros durante la semana y manteniendo su núcleo familiar en el país de origen. Las fluctuaciones en las políticas de inmigración incidieron para que el personal chileno aumentara o disminuyera según esos vaivenes. La inversión social que ya en 1966 era señalada como un factor negativo en los costos de producción, continuó siendo una importante fuente de erogaciones. En 1967 se dicta la ley 17493 por el cual se faculta a la empresa a donar o vender lotes al personal argentino sin embargo esto se concretará recién en la década de 1980 en que YCF inició la venta de viviendas a sus ocupantes, agentes de la empresa. En 1983 se firmaron 330 boletos de compra-venta en Río Turbio y a pesar de esta incipiente desvinculación entre la empresa y el personal, se mantuvieron por algún tiempo los servicios comunitarios, por ejemplo, el servicio cloacal, la provisión de agua corriente y la calefacción del 35\% de las viviendas de la Villa Minera. En relación a las transformaciones producidas en Santa Cruz puede considerarse como positivo el paulatino poblamiento en la zona del yacimiento, aunque la marcada dependencia con el Estado y la creciente crisis de la explotación, muestran una población altamente inestable ante la falta de alternativas económicas, la transformación de la zona orientándola hacia la actividad turística aún no muestra los resultados esperados y la privatización del yacimiento solo ha servido para paliar en parte la crisis laboral, sometiendo a los trabajadores que antes dependían de la empresa estatal a las nuevas condiciones de precarización.

\section{Yacimientos Carboníferos Fiscales, hoy Yacimientos Carboníferos Rio Turbio $(Y C F / Y C R T)$}

Como se mencionó anteriormente, desde finales del siglo XIX se conoció la existencia de carbón en Río Turbio, pero no fue sino hasta los años 30 que Yacimientos Petrolíferos Fiscales (YPF) explora, en búsqueda de petróleo. El 2 de abril de 1941 el Poder Ejecutivo dictó el decreto 87.672 por el que encomendó a YPF la ejecución de los trabajos para establecer la importancia de los yacimientos carboníferos en el país. Para cumplir con este fin se creó el mismo año la División Carbón Mineral, como un sector especializado de la petrolera estatal. Esta división instaló en Río Turbio dos campamentos para realizar reconocimientos geológicos de los mantos, exploraciones, construcciones de piques, socavones y galerías a principio de 1943, que dieron origen a la primera exploración de tipo experimental. Las características de aislamiento y el despoblamiento de la zona, ocupada solo por escasos establecimientos ganaderos incrementaban las dificultades. Los campamentos eran, en un principio precarios, pero se fueron incorporando pabellones, galpones, depósitos y usinas (Bona, 2000). En 1949, se declara de utilidad pública y sujeta a expropiación por razones de "Defensa Nacional y de orden económico" la explotación de la cuenca carbonífera de Río Turbio. En 1945 se creó la Dirección Nacional de Energía, de donde surgió la Dirección Nacional de Combustibles Sólidos Minerales que prosiguió la tarea iniciada por 
YPF, luego Combustibles Sólidos -ENDE- empresa a la que se transfirió el personal y las responsabilidades de la División del Carbón Mineral de YPF, y desde 1958, Yacimientos Carboníferos Fiscales, de acuerdo al sitio web de YCRT. La decisión de iniciar la explotación comercial en función del nivel de los recursos detectados requirió resolver el problema de transportes, el acondicionamiento de la ruta 293 para trasladar el carbón en camiones, el estudio del trazado de un ferrocarril para trasladar la producción hasta un puerto atlántico y la compra de barcos. Estas actividades estuvieron a cargo de las empresas del Estado, que a su vez debieron coordinar con Vialidad Nacional, El Ministerio de Transportes y Ferrocarriles del Estado para que se efectúen los estudios necesarios y la adquisición de equipos y obras de infraestructura necesaria para poner en producción la mina. También debieron hacerse cargo de la mano de obra y del aprovechamiento de la incipiente población que se iba asentando ligada a la actividad. El recorrido del carbón hasta llegar a los centros de consumo constaba de los siguientes pasos: extracción, depuración, transporte ferroviario (o por camiones al inicio), puerto de Río Gallegos y transporte marítimo (Bona, 2000).

En 1951 se inaugura el primer tramo del ramal ferroviario Eva Perón, aunque este recién es finalizado en septiembre y puesto en funcionamiento en noviembre, acarreando el primer cargamento de carbón hasta Río Gallegos por medio de locomotoras a vapor y formaciones de hasta sesenta vagones.

Se construye por ese entonces el Puerto en Río Gallegos. Para realizar las construcciones necesarias de playa de carga y descarga y almacenaje, y viviendas para el personal, se expropiaron los terrenos necesarios. Inicialmente se construyó un muelle de madera provisorio con sus instalaciones mecánicas, que fue ampliado y mejorado concluyendo las obras en 1956. Sin embargo, la necesidad de incrementar los volúmenes de producción por la creciente demanda de carbón, llevaron a plantear, ya en 1959, la posibilidad de extender el trazado $30 \mathrm{~km}$ hasta Punta Loyola. De este modo se trataba de resolver el problema de marea del puerto de Río Gallegos, donde quedaban los barcos apoyados en el lecho en bajamar, imposibilitando que operaran embarcaciones de mayor capacidad. En Punta Loyola, sobre el océano, operarían barcos de ultramar, de hasta 10 mil Tn, para la salida de petróleo, y posteriormente se desvió el ferrocarril para permitir la salida de carbón por ese puerto. Mientras tanto se fueron ampliando y mejorando las instalaciones del Puerto de Río Gallegos, así antes de su traslado a Punta Loyola, el sistema implementado permitía recibir el carbón del ferrocarril efectuando el vuelco de las vagonetas en una tolva. En Río Turbio se daba la exploración, en Buenos Aires funcionaba la Sede Central de la empresa, con funciones de comercialización, laboratorio, financiamiento, etc., en Río Gallegos había personal técnico, administrativo y profesional, además de los trabajadores de la Gerencia de Transporte y Puerto, mientras la explotación funcionaba en Río Turbio. Los sectores que absorbían mayor cantidad de mano de obra eran la División de Explotación y la División Preparación (Bona, 2000).

En 1958 se crea la empresa Yacimientos Carboníferos Fiscales por decreto del Poder Ejecutivo Nacional No. 3686, sustituyendo a Combustibles Sólidos Minerales que hasta ese año se había encargado de la explotación del mineral. De 1977 a 1980 se experimenta una etapa récord de producción, en el transcurso de esos cuatro años es cuando YCF tuvo su actividad más prometedora en toda su historia, con un grupo de técnicos e ingenieros especializados, se logró en 1977, el récord de producción por un total de 1.450 .000 tn de carbón bruto y 750.000 tn de carbón depurado. Promediando, entre el año 1977 y 1980, se extrajeron un total de 3.500 .000 de toneladas brutas de carbón mineral y se realizaron aperturas de nuevos frentes, como así también de la tecnificación del yacimiento. De 1981 a 1990 se da, lo que el sitio web de YCRT, ha llamado como el ocaso de YCF a consecuencia de los reiterados cambios de poder, la aparición de nuevas tecnologías, la desinversión y desvalorización del carbón mineral, situaciones que año con año incrementan, haciendo cada 
vez más difícil continuar con el mismo ritmo de producción de años anteriores. De esta manera se llega a la Privatización de YCF en el año de 1994, estando a cargo de la presidencia de la nación Carlos Menem quien decide otorgar la concesión al empresario Sergio Taselli, cambiando la denominación de YCF y YCRT S.A., volviendo a ser re estatizada en el año 2000 con la anulación del decreto 1034/02, adoptando la figura de intervención. Entre los planes recientes de la empresa, impulsados por el gobierno nacional, se lleva a cabo la construcción de la Mega Usina a carbón en Río Turbio, inaugurándose el primer módulo de la central térmica en el año 2015 (con información del sitio web de YCRT).

Al igual que la empresa YPF, Yacimientos Carboníferos Fiscales llevó a cabo planes de vivienda para sus trabajadores.

\subsection{Marco teórico conceptual}

Esta investigación se enmarca en los paisajes que estudia la Geografía Urbana, en este caso, los barrios. El paisaje del barrio se estudia a través del análisis de los componentes: plano, usos del suelo y edificación en sus distintas etapas de crecimiento. Desde el punto subjetivo se toman elementos de la Geografía de la Percepción (espacio subjetivo). En el caso de aplicar la Geografía de la percepción desde lo teórico y desde lo metodológico, la información para obtener la subjetividad del ciudadano del barrio que se está analizando es a través de una encuesta. Para realizar la encuesta se debe aplicar una técnica de muestreo que garantice la cobertura territorial del o los barrios que pertenecen al área de estudio. El equipo de investigación que dirige la Lic. Cáceres, directora de esta beca, para lograr una respuesta óptima, aplica técnicas de muestreo bietápicos donde es necesario determinar conglomerados. La determinación de distintas unidades barriales obtenidas a partir de la morfología o paisaje urbano de cada barrio, según las etapas de crecimiento, permite determinar conglomerados para la aplicación. En este caso, entonces, se trata de determinar esos conglomerados que permitirán, en una etapa posterior, la aplicación de encuestas y otras técnicas para la realización de investigaciones propias de la Geografía de la Percepción.

Por otro lado, se adopta a la Geografía Histórica para desarrollar aspectos propios de los barrios, tales como sus inicios, la influencia de las actividades económicas en la conformación de los mismos, los pobladores a los que se destinaron las viviendas; utilizando documentación de archivo, ordenanzas y resoluciones que son el soporte de gran parte de este informe.

Teóricamente, la investigación toma elementos de la Geografía Urbana, ya que desarrolla uno de los principales temas de esta rama: el paisaje urbano y sobre todo la estructura interna urbana, reflejando ciertas características de los espacios vividos. Cáceres (2000), afirma que el paisaje urbano es el resultado de componentes formales relacionados con el marco natural, como el tipo de plano, usos del suelo y la construcción. El plano es el resultado de la combinación sobre el espacio de la ciudad a través del tiempo, de superficies libres (calles, parques, jardines, plazas, etc.) y de superficies construidas. Los ejes de fijación naturales (río, bahía, curva de nivel) y humanos (vías férreas, rutas) influyen en la orientación del plano.

Para un mayor entendimiento de los resultados, a continuación, se abordan 3 aspectos necesarios para la comprensión del informe: el primero referido al barrio; el segundo a la estructura interna de la ciudad de Río Gallegos; y el tercero referido a la costanera.

\section{El barrio}

Los usos de suelo, esto es, las diferentes maneras de utilizar el espacio en función de las necesidades y actividades de la población que vive y trabaja en la ciudad (Zárate, 1984) se clasifican en: administrativo, comercial, residencial, industrial. De esta forma, se puede realizar una sectorización de acuerdo al uso de suelo que predomine. Es así que surgen los 
barrios que junto con el hogar o vivienda y la manzana se consideran como unidades constitutivas importantes. Las dos últimas son construcciones delimitadas por vías de circulación, sin tanta complejidad, el barrio, en cambio, es una sectorización de la ciudad más compleja. Un barrio, según Zamorano (1992) está compuesto de islotes y calles, en un conjunto que constituye un medio de vida y de actividades dotado de individualidad... sectores homogéneos, espontánea o planificadamente, cuya superficie tiene un destino específico, ya sea negocios, industria, administración o residencia. Las formas variables, las delimitaciones indecisas, las interpretaciones frecuentes, los diseños alternativamente lineales o espaciales, no anulan la tendencia al agrupamiento, de acuerdo al tipo de relaciones. Asimismo, cuando la superficie correspondiente es circunscrita como espacio administrativo, de gestión, suele suceder que no existe coincidencia con el barrio, en cuanto éste implica una vivencia especial para los ciudadanos. El habitante de los barrios residenciales se enfrenta por un lado y aprecia por el otro, a circunstancias agradables o desagradables que parten de una falta de sentido comunitario y de condiciones de vida atractivas.

- Barrio Residencial: ocupan la mayor superficie de las ciudades y está en relación directa con la disociación entre el trabajo y el lugar en el que se habita. Deben contar con equipamiento para satisfacer las necesidades de los habitantes como comercios, escuelas, gimnasio, centro de salud, etc. Las condiciones socioeconómicas de sus habitantes se evidencian en su fisonomía, en los materiales de construcción, en las formas de apropiación de los terrenos en cuanto al tamaño y proximidad de las casas, en la infraestructura vial y de servicios, en la disponibilidad de transporte, etc.

- Barrio Administrativo: Se concentran los edificios de los servicios públicos y gubernamentales.

- Barrio Industrial: Se concentran las actividades industriales, suelen ser planificados y estar en las afueras de la ciudad sobre importantes ejes de comunicación.

- Barrio Comercial: se caracteriza por la concentración de establecimientos comerciales. La construcción se da por la tipología de los edificios y el volumen de la edificación. En este sentido, los centros urbanos surgidos en Santa Cruz el siglo pasado, eran reflejo de la vivienda rural, el material de construcción era de chapa con techo a dos aguas, de una sola planta. Las corrientes migratorias de los años 60's y 70's incorporan el cemento. Y en las últimas décadas se ha rescatado el techo a dos aguas. Predominan las viviendas de tipo unifamiliar, adosadas unas a otras. Poco a poco se ha ido incorporando el árbol y el cuidado del jardín. En los últimos años se han construido departamentos dúplex en lotes muy angostos. Cufré y Zonaro (1985), especifican que la vivienda no es una creación de tipo local o regional, sino que son tipos importados, que se fueron adaptando a las condiciones particulares. Ante el aislamiento y la distancia de los centros de poder la única alternativa posible fue la construcción en seco o prefabricada, ya que la falta de materiales producidos en el marco local impidió o dificultó, por excesivamente costosa, la construcción con materiales tradicionales, como se efectuaba en el resto del país. Esta situación determina la principal característica de la tipología de la vivienda patagónica.

\section{Estructura interna de Río Gallegos}

Río Gallegos es una ciudad intermedia de la Patagonia. De acuerdo con Cáceres y García (2004), en su estructura interna se pueden distinguir diversas áreas que han surgido en distintas etapas, atendiendo a múltiples factores.

1. Área Central.- Se caracteriza por la traza de un plano en damero a partir de dos ejes perpendiculares: las avenidas Kirchner (antes Roca) y San Martín. Constituye el espacio más dinámico de la planta urbana. Las calles son amplias, de 25 metros de ancho para un mejor aprovechamiento de los rayos solares. En esta área se distinguen 
el centro y el pericentro. El centro coincide con la primera traza de la ciudad, que es también el sector histórico, predominan las funciones administrativas (de la gestión nacional, provincial y municipal), concentra las principales actividades, el mayor equipamiento y el área de influencia dentro de la red urbana. El pericentro es el área intermedia entre el centro y las áreas residenciales, corresponde con el ensanche de la planta urbana en 1924, también se localizan edificios públicos, comercios, prestadores de servicios, centros recreativos y centros médicos.

2. Segunda Franja Residencial.- Crecimiento de la ciudad a partir de las década de los 60 y 70 debido no solo a la actividad ganadera y del frigorífico, sino también al asentamiento de la administración de YCF, de los campamentos de YPF y de la administración pública.

3. Tercera Franja Residencial.- Corresponden a las áreas que se consolidaron en la década de los 90’s, aprovechando el cierre del ramal ferro industrial Río Turbio y del muelle homónimo, y las transformaciones que se fueron dando en el borde urbano (Cáceres, et.al., 2013).

\section{La Costanera, el Puerto y el Ferrocarril}

La margen sur de la desembocadura del río Gallegos permitió, entre otros, la construcción de la costanera. La misma resulta importante puesto que el área de estudio abarca una parte de ella. Cáceres y García en 2003, definen tres etapas de crecimiento en Río Gallegos entre los siglos XIX y XX cuyos cortes temporales responden a hechos económicos, políticos sociales que provocaron ritmos de crecimiento de la población que se refleja en el paisaje urbano: la Provincialización del Territorio de Santa Cruz, creación de YPF e YCF, traspaso a YCRT y ampliación de la trama urbana en tierras de las Fuerzas Armadas. Estas fases asociadas a las áreas internas de la ciudad aplicados a la costanera dan como resultado lo siguiente:

Primera etapa: comienza en 1885 con el surgimiento de la ciudad; pertenecen al Área Central. Las construcciones se caracterizan por tener rasgos de arquitectura pionera, mirando hacia el norte para aprovechar la luz solar.

Segunda etapa, comienza en 1957 con la provincialización del Territorio de Santa Cruz y la creación de YPF e YCF que corresponde con la Segunda Franja Residencial y en la actualidad son los tramos occidental y oriental comprendidos entre las calles Rawson - Av. Balbín (ex Vías del FFCC) y Sureda - Malaspina, respectivamente. En el tramo occidental de la Costanera la presencia de YCF condiciona el paisaje urbano, es decir, la actividad extractiva del carbón de Río Turbio a través de los usos del suelo y las construcciones materiales y estilos arquitectónicos propios de la morfología urbana rioturbiense. La presencia del Puerto Río Gallegos, para la salida por vía marítima del mineral, el ferrocarril de Trocha Industrial, las instalaciones de chapa tipo mediocaño (quonset), montículos de carbón y unas pocas viviendas con paredes de quebracho. Así mismo la llegada de YPF es visible a través de los tanques de almacenamiento de subproductos del petróleo y luego con la construcción del Barrio Náutico.

La tercera etapa, es a partir de la década del 90 con la privatización de YCF e YPF, y la ampliación de la trama urbana en tierras de las Fuerzas Armadas. A fines del siglo XX, el Puerto Río Gallegos es trasladado al Paraje Punta Loyola y permite la continuidad de la Costanera hacia el tramo occidental.

Consideraciones a tomar en cuenta:

- Teniendo, los elementos indispensables para poder interpretar los resultados, es necesario recordar que las manzanas identificadas en los resultados, son aquéllas que fueron construidas por la empresa YCF e YCRT. Esta identificación se hizo bajo un 
enfoque histórico, basado en entrevistas y documentos encontrados en el archivo municipal y en la oficina de catastro de Río Gallegos, entre otros.

- Se puede reconocer en el paisaje urbano, características propias de la geografía industrial, ya que a estos barrios residenciales, sobre todo a los localizados en el área central, se los puede relacionar con la actividad carbonífera, producto de los elementos que allí se encuentran apostados. Los galpones, depósitos, vagones de los trenes que hoy se encuentran abandonados, rememoran la actividad económica extractiva desarrollada en la Cuenca Carbonífera, que veía en Rio Gallegos el lugar al cual llegaba la producción para ser embarcada y traslada a su destino final.

Otros conceptos de interés:

Ejes estructuradores. Se refiere a las calles, que dada su importancia se han clasificado en ejes estructuradores de primer orden, segundo orden y tercer orden. Es un término usado en la cartografía.

Mensura. Se entenderá como la determinación, medición, ubicación y documentación en un plano de los inmuebles y sus límites conforme a las causas jurídicas que los originan, es decir, la aplicación del título de propiedad al terreno propiamente dicho. La mensura es la generadora de la parcela catastral. El documento que la contiene se llama Plano de Mensura. (Catastro, Provincia de Santa Fe), y es un documento legal, técnico y administrativo. En los planos de mensura, analizados en el proceso de investigación, contienen los siguientes datos:

- Título (que a veces aparece con la palabra objeto), refiere a la mensura de la manzana, incluida la fracción y circunscripción. Puede ser que sea mensura o redistribución de mensura.

- Nombre del propietario, ya sea particular, empresa pública o privada, o cualquier otra asociación u organismo que ha adquirido un bien.

- Antecedentes, se refiere a los planos de mensura anteriores.

- Ubicación: va escrita y acompañada de un croquis.

- Firma del propietario, que no siempre aparece.

- Firma del agrimensor.

- Varios sellos, generalmente el sello de "aprobado", de fecha de inscripción en la Dirección de Catastro, sello de la Municipalidad, etc.

- Número de identificación. Generalmente aparece un número precedido por una $\mathrm{M}$, o bien un número de plano. Aunque hay ocasiones en que no se observa ninguno.

Parcela catastral. Es la unidad catastral, es decir, el bien inmueble urbano, suburbano o rural designado como lote, fracción o unidad de propiedad horizontal, en un plano de mensura inscripto en el organismo catastral competente. (Catastro, Provincia de Santa Fe). Este concepto generalmente no aparece en los documentos oficiales, es más común leer la palabra lote o fracción. Sin embargo a veces se hará uso en los resultados.

Solar. En la modalidad de mensura catastral adoptada en Río Gallegos, un solar se refiere a cada una de las 4 partes en las que se puede dividir una manzana, generalmente se identifican con las letras a, b, c y d.

YPF. Yacimientos Petrolíferos Fiscales. Empresa encargada de la exploración, explotación, comercialización, etc., del petróleo en la República Argentina.

YCF. Yacimientos Carboníferos Fiscales. Empresa estatal dedicada a la extracción del carbón.

YCRT. Yacimientos Carboníferos Río Turbio.

SIG. Sistema de Información Geográfico. Programas creados con la finalidad de procesar información geográfica. 


\subsection{Marco legal}

- A nivel nacional: Ley Orgánica de los Territorios Nacionales 1884; Ley N ${ }^{\circ} 14$ 408, Sobre la Provincialización de los Territorios Nacionales; Ley 12966, declaración de expropiación de la cuenca carbonífera. Año 1949.

- Relacionados a YCF/YCRT: Decreto 87672, facilita la exploración de YPF en la zona de Río Turbio; Decreto 3686, creación de YCF; Ley 17493, facilita a la empresa YCF a vender o donar los lotes de su propiedad a empleados argentinos; Decreto 1034/02, sobre la reestatización de YCRT.

- A nivel provincial: Ley $N^{\circ} 1$ 532, Creación del Territorio de Santa Cruz, 1884; Decreto de Creación de la Subprefectura Marítima de Río Gallegos, 1885; Ley de traslado de la capital de Santa Cruz a Río Gallegos, año 1898.

- A nivel municipal: Decreto 510, sobre límites de barrios. Año 1977; Ordenanza 2949-HCD01, sobre designación del Barrio YCF 2001; Carpeta de calles, legislación vigente, listado, Honorable Concejo Deliberante.

- Del Archivo Histórico Municipal: Exp. N 1419, año 1977, iniciado por el Departamento ejecutivo, "Límites de los Barrios de nuestra ciudad".

- Sobre aprobación de planos para construcción de viviendas de YCF en el archivo municipal: Expediente No.12312, de 1974; Expediente No.12313, de 1974; Expediente No.12314, de 1974.

- Planos de Mensura de YCF de la Dirección de Catastro: Zona a reservar para Proyecto de obras portuarias e instalaciones para embarque de carbón, No.1793 D.G.C-P-1, de 1950; Mensura oficial con deslinde de las fracciones II y III, decreto 1250/72, Exp. 3900/YCF/72, año de 1972; Mensura de la fracción I que comprende parte de las manzanas 1. 2. 3. 4. 14, 15, 16, 17, 23, 24, 31, 32 y 283 y tierras del sector nord-oeste, Decreto 1024/71. Exp. 5991/Y/71, año 1971; Mensura oficial de la manzana No. 14, sección "D", Circunscripción III, Plano 34D-1976, año 1971; Mensura oficial con subdivisión de la manzana "14", sección "D", Circunscripción "III", Plano 91-P-76, año 1976; Mensura oficial de la manzana "24", sección “D”, Circunscripción "III", Decreto 007/76, Exp. 2537/YCF/76 de 1976; Mensura oficial con subdivisión de la manzana "24", sección "D”, Circunscripción "III", Decreto 694/76, Exp. 5348/Y/76 de 1976; Mensura administrativa con subdivisión de las manzanas No. 42 "A" y 42 "B", M3028, de 1987; Mensura oficial con subdivisión y nueva subdivisión de la manzana “44", sección "A" Plano No. 013-M-1976, Decreto 369/76, Exp. 1260/Y/76, de 1976; Mensura administrativa con subdivisión de las manzanas No. 43-44-47-62, sección "A", Circunscripción "I", Plano No. 117-M-1975, Decreto 1033/75, Exp. 3565/Y/75 de 1975; Mensura oficial con fraccionamiento de las manzanas No. 64 y 65, Decreto 773/71, Exp. 4985/YCF/71 de 1971; Mensura con redistribución predial manzana 65; Decreto 1898/74, Exp. 8010/YCF/74, de 1974; Mensura con redistribución predial manzana No. 80, Decreto 1999/74, Exp. 8012/YCF/74, de 1974; Mensura con redistribución predial manzana 18. Sección A, Circunscripción I, Decreto 734/71, Exp. 4989/YCF/71, de 1972; Mensura y división Manzana 81, M3465, de 1988; Mensura con redistribución predial manzana 101, Decreto 1927/74, Exp. 8045/YCF/74 de 1974.

- De la Dirección de Informática de la Municipalidad de Río Gallegos: Se obtuvieron diversos instrumentos legales, que para preservar la ubicación de los predios no se pondrán en esta sección. Sin embargo, algunos fueron mencionados en los resultados. 


\section{RESULTADOS, ANÁLISIS Y DISCUSIÓN}

\subsection{Materiales y Método}

Los materiales utilizados son: bibliografía en libros y artículos científicos de forma física papel o digital; instrumentos obtenidos en las distintas dependencias públicas; diarios de circulación local; elementos de librería; computadora, impresora e insumos de impresión; software para procesamiento de datos; automóvil; cámara fotográfica; celular smartphone; Plano catastral de la ciudad de Río Gallegos.

La investigación comenzó con la delimitación del tema y área de estudio, así como también los objetivos, trazando un plan de trabajo. En primer lugar se hizo un relevamiento bibliográfico en fuentes de información al alcance: biblioteca de la UNPA, virtual y personal para conocer la información disponible del tema, y también para la ampliación del marco teórico y conceptual. Se recabó información sobre la historia de Río Gallegos, Río Turbio, YCF-YCRT, etc. Posteriormente se dio lectura a la bibliografía seleccionada y se realizó una primera salida de campo para reconocer el área de estudio. En segundo lugar, se acudió al Archivo Histórico Municipal, Catastro y la Dirección de Informática del Municipio de Río Gallegos para localizar los primeros planos de mensura de terrenos cedidos a YCF-YCRT. En tercer lugar, se localizaron a personas que son referentes de YCF-YCRT, Sres. Luis Peralta que brindó las primeras pistas de ubicación de las viviendas, y Luis Héctor González, quien aportó información valiosa sobre las viviendas de la $3^{\circ}$ y $4^{\circ}$ etapa de construcción, entre otros. En cuarto lugar, se recorrieron las manzanas sobre las cuales ya se tenía información a fin de realizar la observación directa y detectar otras posibles viviendas, por similitud. Además, se analizaron imágenes satelitales del área. En esta etapa se tomaron fotografías del barrio. En quinto lugar, se acude a los SIG para registrar los primeros datos. Se vuelve a acudir a Catastro Municipal para seguir obteniendo más información. Y por último, se realiza la cartografía que acompaña este informe y se realiza el informe propiamente dicho.

\subsection{Resultados y análisis}

El área en estudio se localiza al noroeste del centro de Río Gallegos. Las manzanas comprendidas están en el barrio conocido por la municipalidad como "Barrio El Trencito" a excepción de 2 que se encuentran en el Barrio YCF.

El Barrio El Trencito, está delimitado por la Avda. Almirante Brown (Avda. Costanera) al norte, Ricardo Balbín (ex vías férreas) al oeste, y Pdte. Néstor Kirchner (ex Julio A. Roca) al sur. El límite oriental está definido por las calles Federico Sphur, Avellaneda y 9 de Julio. Limita con los barrios El Puerto, al sur; Zona Centro, al sureste; Evita al suroeste; e YCF al oeste. El norte corresponde al espacio público abierto Costanera debido a la presencia del estuario del río Gallegos (figura 2). En la actualidad el uso está destinado a la recreación y el ocio, en el pasado a la presencia del puerto carbonero hasta donde llegaba el ramal ferroviario que transportaba el carbón extraído en el yacimiento Río Turbio y que era trasbordado desde los vagones a los barcos, algunas de las instalaciones están deterioradas, y son vestigios de la infraestructura de dicho proceso (Figura 3 ).

Las instalaciones portuarias, donde se destaca aún el Muelle El Turbio, comenzaron a proyectarse desde el año 1950 por el Ministerio de Obras Públicas de la Nación, bajo la Dirección Nacional de Construcciones Portuarias y Vías Navegables (figura 4, foto a), siendo el iniciador Yacimientos Petrolíferos Fiscales (YPF), según expediente $73840^{3}$ bajo el

\footnotetext{
${ }^{2}$ Según Decreto 510 de la Municipalidad de Río Gallegos, con fecha del 16 de marzo de 1977.

${ }^{3}$ El plano en dicho expediente, corresponde al Expediente 6113-950 del Ministerio de Agricultura.
} 
extracto: "s/reserva terreno en Río Gallegos p/construcción instalaciones portuarias destinadas al embarque del carbón de Río Turbio". En el plano se observa la dimensión de obra para lo cual se necesitaba una amplia extensión de terrenos para su operabilidad. De esta forma, se puede entender cómo nace una cierta dinámica al noroeste de la ciudad. Al construirse el puerto a principios de la década de 1950, se deduce que esos terrenos fueron cedidos a YPF originalmente, y posteriormente pasan a control de YCF al crearse esta empresa en el año de 1958. Esta deducción se refuerza al analizar un plano de mensura del año de 1971, que tiene por objeto la "Mensura de la Fracción I de Y.C.F que comprende parte de las manzanas 1, 2, $3,4,14,15,16,17,23,24,31,32$ y 283 y tierras del sector nord-oeste" siendo propietario YCF (figura 4 , foto $b$ ).

Estas manzanas abarcan gran parte del plano anterior que trata sobre la construcción del puerto mismas que a partir de la década de 1950 se han poblado poco a poco, de distintas maneras, por ejemplo, a través de la venta directa de manzanas y parcelas a particulares o bien a través de planes de vivienda impulsadas por la empresa estatal YCF/YCRT para sus trabajadores.

$\mathrm{Al}$ tener, entonces, como base todo lo anterior, y al reunir los testimonios recopilados durante el proceso de investigación las manzanas $(\mathrm{Mz})$ residenciales que dan lugar a este informe reúnen tres criterios esenciales:

1) Tienen un vínculo con la empresa Yacimientos Carboníferos Fiscales, hoy Yacimientos Carboníferos Río Turbio (en adelante YCF, YCRT).

2) Son manzanas residenciales parcial o totalmente.

3) Han sido mencionados en las entrevistas o testimonios recabados, como parte de los planes de construcción de viviendas que ha ejecutado la empresa YCF/YCRT en distintas etapas.

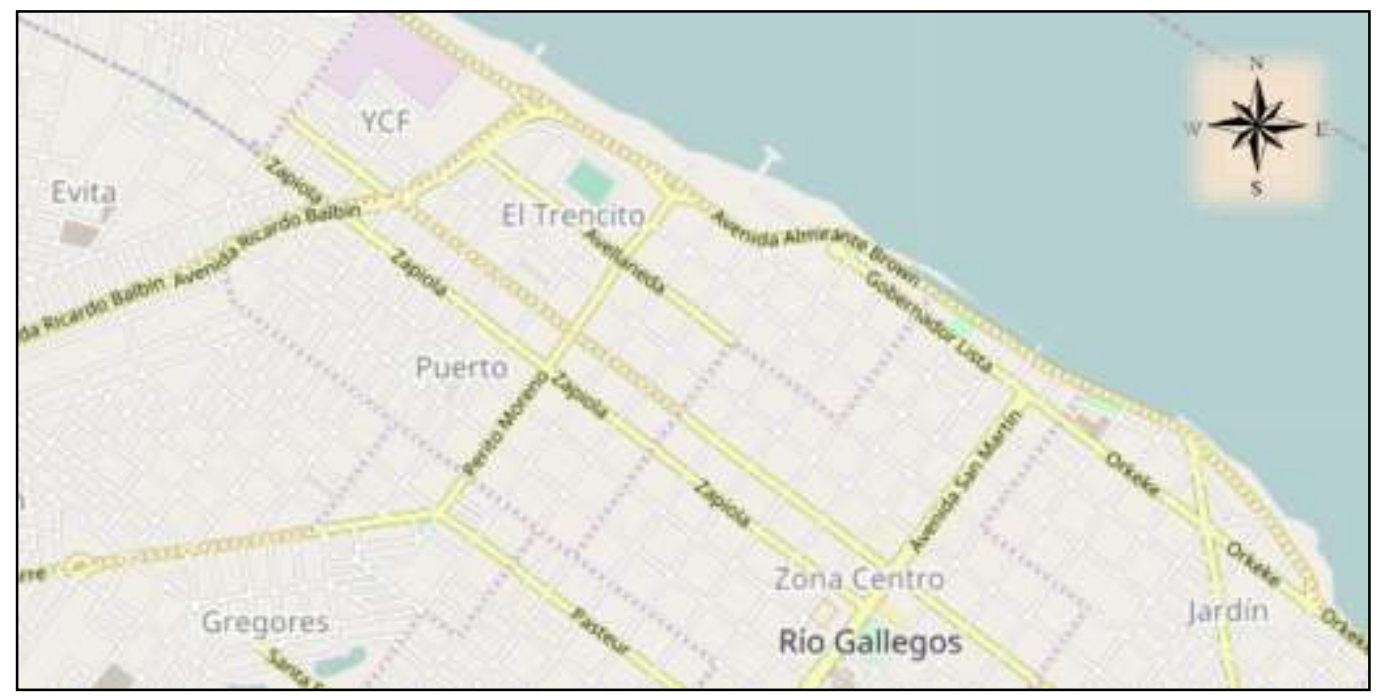

Figura 2. Ubicación Barrio El Trencito, con límites establecidos por decreto 510.

Fuente: OpenStreetMap $200 \mathrm{mts}$.

Esta selección de manzanas da como resultado que la empresa YCF/YCRT ha construido viviendas para sus empleados en 4 etapas cronológicas diferentes: la primera en la década de 1950 en dos manzanas; la segunda se da en la década de 1970 en 6 manzanas; la tercera en la década de 1980 en 4 manzanas; y la cuarta entre el 2005 y 2013 en dos manzanas (figura 14). 


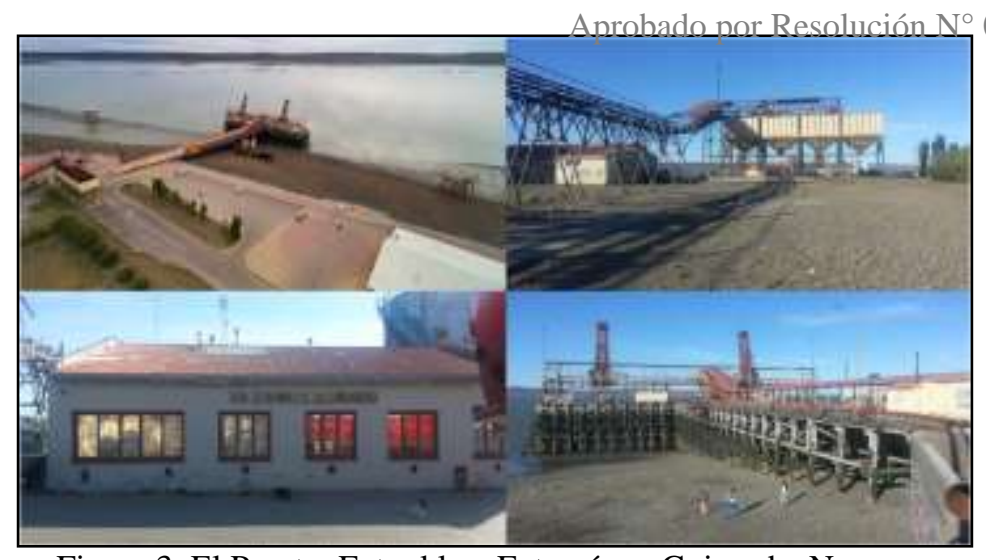

Figura 3. El Puerto. Foto: klap. Foto aérea: Guisande, N.

Etapa 1: Comprende los primeros años de la década de 1950. Fueron construidas por YCF como empresa, incluso con sus propios empleados, sobre las manzanas más próximas al muelle. Aquí se concentran las casas de durmientes ${ }^{4}$, que son un distintivo visual que las destaca de las viviendas que las rodean.

Las viviendas están construidas en las Mz. 80, 81A Y 81B entre las calles de Avellaneda, Sebastián Elcano, Avda. Almirante Brown y Mendoza.

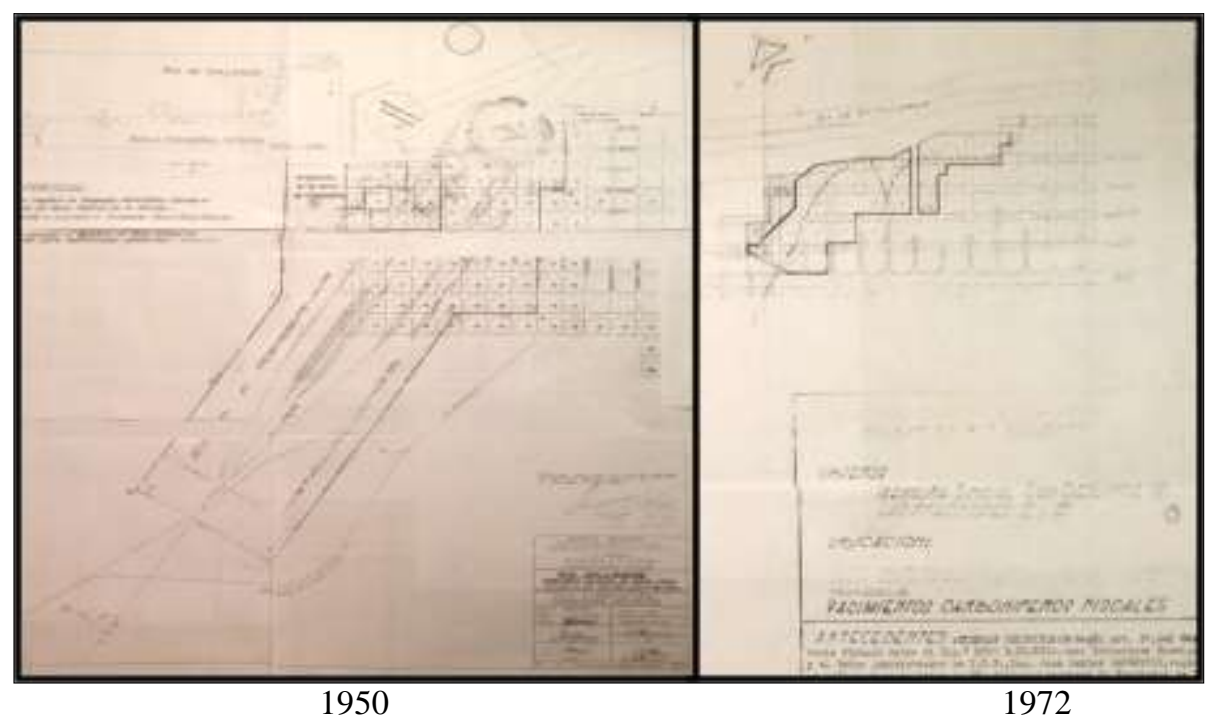

Figura 4. Plano para instalación del puerto VS Manzanas propiedad de YCF

Mz. 80.- Se encuentra entre las calles Pellegrini, Elcano, Avellaneda y Mendoza. Está dividida por dos pasajes que convergen al centro de la manzana, uno es el pasaje Adolfo Wild por calle Mendoza y el otro, el pasaje Pablo Nieva por calle Pellegrini, resultando 2 polígonos de diferente tamaño, así consta en la "Mensura con redistribución predial manzana 80 " del año 1974, con antecedente de mensura aprobada por decreto 925/70 en el expediente 3114/YCF/70, del año 70. En el polígono más pequeño, se observan 4 casas de durmientes (sobre la calle Mendoza y Pellegrini) de las 12 viviendas que hay. En el polígono mayor, sobre calle Elcano se asientan las oficinas de YCRT, y sobre calle Avellaneda el Ministerio de Producción (figura 15), edificio que anteriormente albergó a las oficinas centrales de $\mathrm{YCF}^{5}$. El

\footnotetext{
${ }^{4}$ Los durmientes son, en vías férreas, los elementos transversales al eje de la vía que sirven para mantener unidos y a la vez a una distancia fija (galga o trocha) a los dos carriles (rieles) trasmitiendo el peso del material rodante al balasto y, por intermedio de éste, al suelo. También cumplen la función de dar peso al conjunto, de manera que la geometría inicial del trazado se mantenga en la mayor medida posible. Se fabrican de diversos materiales, entre ellos madera, hierro y hormigón (Wikipedia, concepto revisado por el Ing. Gerardo Riesco).

5 Actualmente las Oficinas de YCRT en Río Gallegos están en Elcano 242, y la planta de operaciones en Punta Loyola.
} 
resto son 14 viviendas. Cabe mencionar que las casas donde se asientan las oficinas de YCRT y las aledañas (que no son de durmientes) fueron construidas con madera que se trajo de los bosques de Río Turbio. En las casas construidas entre Nieva y Wild, vivía el personal jerárquicos dentro de la empresa o los que eran necesarios para el funcionamiento de las instalaciones, por ejemplo de la luz, el puerto, etc. El nombre de los pasajes Wild y Nieva corresponden a 2 agentes de la empresa que fueron clave en el funcionamiento del puerto, del tren y también para la construcción de las viviendas.

Actualmente viven, en algunas de ellas, sus hijos o nietos. En otros testimonios se supo que en algunas de estas casas también funcionaban a veces como gamelas para los trabajadores que venían de fuera, principalmente de Río Turbio.

Mz. 81 (A y B). Está entre las calles Avda. Almirante Brown, Elcano, Pellegrini y Mendoza. El primer registro encontrado sobre esta manzana, obedece a una "Mensura con Redistribución Predial Manzana 81" del año de 1972, siendo propiedad de YCF, con un antecedente de mensura aprobado en 1971. En el mismo figura sin división y con inscripción en la Municipalidad de Río Gallegos desde el 25 de septiembre de 1944. El segundo registro catastral encontrado se da en la "Mensura y división, de la manzana 81" en 1988, propiedad de YCF, con el número M3465, que figura con la división de manzana en dos secciones. El pasaje José Lasalle divide a la manzana en la sección A que es la más próxima al muelle y la sección B.

En la sección A, se reconocen 10 viviendas de las cuales solo 2 aún conservan su fachada de casa de durmientes. Por la calle de Elcano se ubica la capilla "Virgen Niña" y por la calle Mendoza se encuentra la Asociación Centro de Jubilados, Pensionados y Retirados de YCF (figura 15). Cabe mencionar que esta sección solo está ocupada la mitad que da hacia el pasaje Lasalle. La mitad que da hacia la costanera, no tiene edificaciones pero pertenece a YCF.

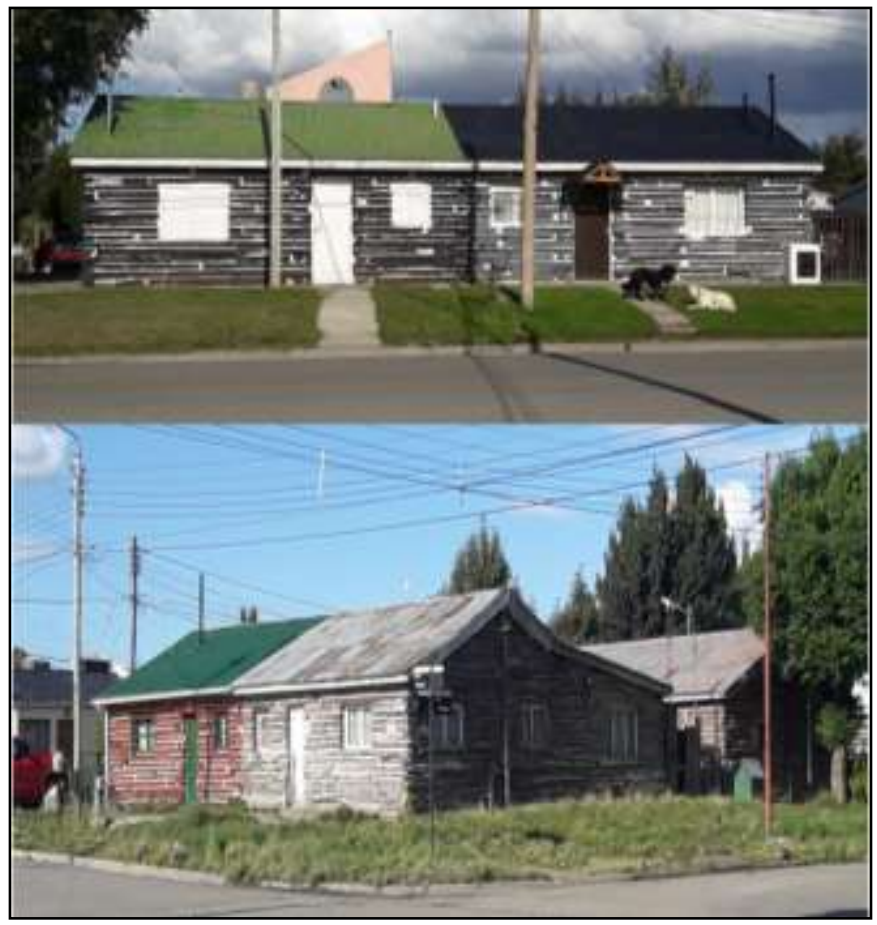

Figura 5. Casas fabricadas con durmientes. Foto: klap 
En la sección B, se observan 16 casas de las cuales 10 se reconocen casas de durmientes (figura 5). Varias han sido modificadas con ampliación y no se observan construcciones en primer piso.

Uno de los entrevistados comentó que en el año de 1952 se construye el Gimnasio del Club Ferro con los durmientes, hoy este gimnasio ya no existe, pero para aprovechar los durmientes deciden construir las casas. Revela también que su construcción fue un trabajo "hasta artesano" porque ponían el durmiente, después una especie de barro, luego una línea finita de cemento y enseguida el otro durmiente, que a veces venía deformado. Las casas constan de una cocina y dos dormitorios y en algunas se puede ver los durmientes deformados. La disponibilidad de durmientes se debía porque a la par de la construcción del puerto, se estaban haciendo las vías férreas para el ramal industrial Río Turbio - Río Gallegos.

Se ha mencionado a la Capilla Virgen Niña, según testimonios data de esta época y forma parte de la identidad del barrio, incluso ha sido reconocida como patrimonio de la ciudad, según una nota del 20 de marzo del 2010 del diario TiempoSur donde, además, se lee: "esta capilla la elegimos por la historia que tiene, la cual se encuentra en un barrio muy conocido e importante de la ciudad para el sostén espiritual de las familias de YCF, (...) faltaban padres y venían desde río Turbio para dar las misas en donde todo el polo obrero-carbonero de esta ciudad se acercaba a ella ya que vivía en ese barrio."

\section{Etapa 2.}

Corresponde a la década de 1970 Fueron construidas por YCF como empresa. Se encuentran ubicadas alrededor de la cancha del Club Ferro. Corresponden a las manzanas 43B, 44, 47, 62B, 65B que se encuentran entre las calles de Lasalle, La mañana, Wild, Teniente de Castillo, Pellegrini, Mendoza, Avellaneda, Fitz Roy Alberdi y Alberti. Y la manzana 101 entre las calles Gobernador Moyano, Federico Sphur, Pellegrini y Córdoba. Esta última manzana de acuerdo a recuerdos de vecinos se hizo la ubicación en esta etapa.

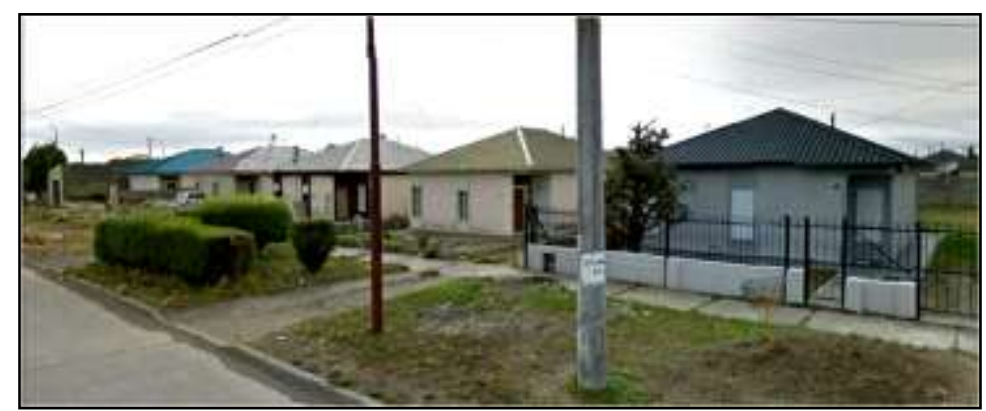

Figura 6. Modelo de casas, etapa 2, se caracteriza por el techo cuadrado. Foto: Google Earth.

Mz. 43 (mitad norte de la sección B). Ubicada entre las calles Avellaneda, Fitz Roy, Alberdi y Alberti. Actualmente está dividida, por la calle Saint Exupery, en dos secciones A y B. El registro catastral es de una Mensura Administrativa con Subdivisión de las Manzanas No. 43, 44, 47 y 62, propiedad de YCF, con fecha de inscripción en el Registro de Subdivisiones del municipio del 3 de Junio de 1975. Sin embargo, en 1974, YCF, había iniciado una solicitud de aprobación de planos de construcción de viviendas para esta manzana, así consta en el Archivo Municipal, Expte. No. 12312. En este registro se aprecian solo 5 lotes y ninguna división de manzana, que al comparar este croquis con la disposición actual de las viviendas, se deduce que se trata del complejo de viviendas en la mitad norte de la Sección B, se trata de 5 viviendas de las cuales solo se reconocen 3 en su prototipo original (figura 6). La otra mitad de esta sección está ocupada por un establecimiento público. Con respecto a la sección A, se construyó a finales de los años 80 , se retomará en la etapa 3 . 
Mz. 44. Delimitada por las calles de Lasalle, Fitz Roy y Avellaneda. Por el Expte. No. 12313 del año de 1974, encontrado en el Archivo Municipal, se sabe que YCF realizó la solicitud de aprobación de planos para construcción de vivienda en esta manzana. Dos años más tarde, se hace la "Mensura Oficial con unificación y nueva subdivisión", así consta en el Plano No. 013-M-1976, con fecha de inscripción en el registro de subdivisiones de 3 de marzo de 1976. El plano de la manzana presentado en el Expediente No. 12313 coincide en número y disposición actual de los lotes. Se trata de 11 viviendas de una sola planta, dispuestas sobre la calle Fitz Roy y Avellaneda, sin edificación el resto de la manzana, cuyo baldío es visible desde la calle Balbín. Una particularidad de esta manzana es que la mayoría se conserva con su forma original (figura 6) y por el escaso arbolado, son distinguibles por el color de su techo cuadrado.

Mz. 47. Se ubica entre las calles Lasalle, La Mañana, Avellaneda, y Fitz Roy. Los registros más antiguos en el Archivo Municipal, son 3 expedientes del año 1974, donde YCF solicita la aprobación de los planos de vivienda a construir: Expdte No. 12312 que contiene 4 lotes que dan hacia la calle Fitz Roy; Expdte. No. 12313 con 3 lotes sobre la calle Lasalle; Expdte. 12314 con los 20 lotes restantes que complementan la manzana. También se da cuenta de esta manzana en el Plano No. 117-1-1975 que trata de la "Mensura administrativa con subdivisión de las manzanas 43, 44, 47 y 62," propiedad de YCF. Es una manzana con un total de 27 viviendas, todas conservando el característico techo cuadrado de su diseño original (figura 6). La disposición actual de los lotes coincide con los expedientes del Archivo Municipal.

Mz. 62B. Se encuentra entre las calles Wild, Teniente del Castillo, Avellaneda y La Mañana. El único registro que se obtuvo de ella fue el Plano No. 117-1-1975 que trata de una Mensura Administrativa con subdivisión, propiedad de YCF. Esta manzana se incluye por analogía, puesto que de las 15 viviendas que existen, 10 conservan el característico techo cuadrado (figura 6) y la mayoría está sobre la calle Avellaneda. Cabe destacar que esta es una manzana angosta, la mayoría de las viviendas ya han sido modificadas. La calle Wild la divide de la Mz. 62A, ocupada en su totalidad por las instalaciones del Club Social y Deportivo Ferrocarril YCF quien es propietario.

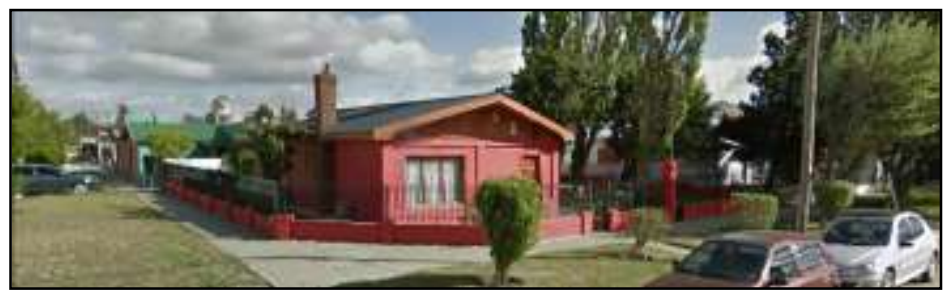

Figura 7. Casas de la manzana 65. Foto: Google Earth.

Mz 65. Está entre las calles Pellegrini, Mendoza, Avellaneda y Tte. De Castillo. El primer registro catastral que se encuentra es la "Mensura Oficial con fraccionamiento manzanas No.64 y 65, del año 1971, con antecedentes de 1969. Posteriormente, figura en la "Mensura con redistribución predial manzana 65" del año 1974. Ambas mensuras mencionan a YCF como propietario. Por otro lado se encontró en el Archivo Municipal el expediente No. 12312 con año 1974, donde YCF pide la aprobación de planos de viviendas para construcción. En este documento aparece una manzana dividida en 5 lotes de tamaño irregular que no se corresponden con la división actual, ya que hoy se observan alrededor de más de 10 viviendas, con distinto tipo de edificación. La mensura de redistribución se puede entender al analizar la manzana actual puesto que si se divide imaginariamente la manzana en 4 solares, en la fracción noroeste se encuentra una cancha pequeña de fútbol (figura 15), que de acuerdo a las entrevistas realizadas, es propiedad de YCRT, y está separada del resto de las viviendas por un pasaje que parte de Pellegrini y llega al corazón de manzana. En el mismo expediente, se encuentran las descripciones de la construcción donde dice que todas son viviendas 
familiares nuevas. Llama la atención que dentro de los prototipos de vivienda a construir, que figura en el expediente del Archivo Municipal, haya viviendas con hasta 4 dormitorios, cocina, star-comedor, y un baño, de una sola planta. Estas descripciones coinciden con las casas sobre la calle Mendoza, que son amplias. Entre las conversaciones con los vecinos, éstos afirman que estas casas eran para el personal jerárquico de la empresa (figura 7).

Mz. 101. Ubicada sobre las calles Moyano, Federico Sphur, Pellegrini y Córdoba. Está dividida catastralmente en 4 solares: A, B, C, D, y es propietario actualmente YCF de los solares A y B, según Mensura con Distribución Predial del año 1974, y antecedentes en el expediente 2788/YCF/70, del año 1970. Se compone de 12 viviendas que se distinguen aún por su prototipo original de una sola planta, aunque varias ya han sido modificadas. Entre el Solar A y C se encuentra un pasaje que empieza en Moyano y termina en el corazón de la manzana. Dadas las observaciones se puede afirmar que la disposición actual de las casas corresponde casi en su totalidad al plano de mensura mencionado, a excepción de una casa más sobre la calle córdoba, y un lote baldío sobre la calle Pellegrini.

Según algunos testimonios, estas casas fueron construidas en los años de 1960, y otros las datan a principios de la década de 1980. Su estructura edilicia parece ser de finales de los setenta (figura 8). También se mencionó que hasta esa altura de la calle Moyano, había un terraplén a donde llegaba el tren a descargar el carbón, incluso mencionan que había otras casas que fueron levantadas cuando sacaron las vías y se construyó la costanera en la década de 1990.

Las viviendas del Barrio Jardín. Dentro de esta etapa, se pueden ubicar unas viviendas de YCF en el barrio Jardín, las de mayor valor inmobiliario por su ubicación, se encuentran en la Manzana 231B, entre las calles Gobernador Mayer, Villarino, Mitre y Cabral.

Esta manzana está compartida con la Caja de Previsión Social de la Provincia de Santa Cruz e YPF. Las viviendas se usaban para el personal de mayor jerarquía dentro de la empresa. El plano catastral de la manzana 231A que es similar pero cedida a YPF, consta de 14 viviendas. Actualmente solo una de ellas sigue perteneciendo a YCF, y el resto fueron vendidas (figura 9).

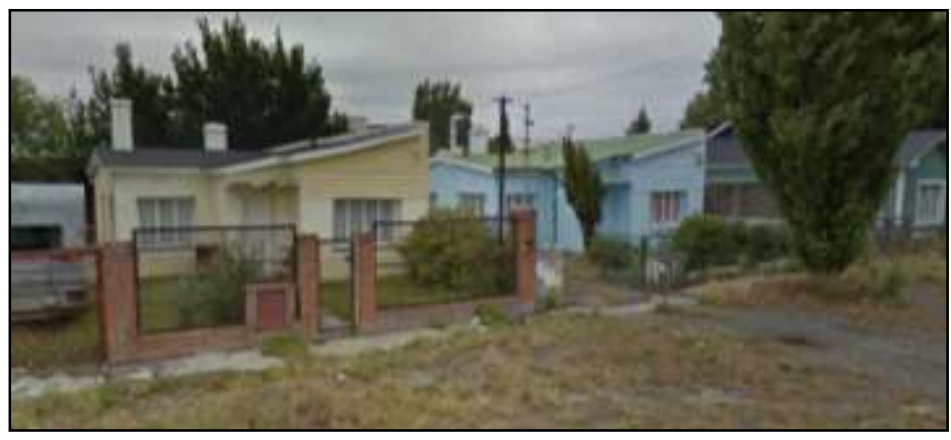

Figura 8. Casas de la manzana 101. Foto: Google Earth

\section{Etapa 3}

Surgen a finales de la década de 19800, a partir de un plan de 200 viviendas individuales, a través del FONAVI, que se consiguen a partir de la formación de cooperativas como la Cooperativa de Consumo y Vivienda Patagónica Limitada. También participaron una cooperativa al interior de YCF, La Asociación Mutual de Trabajadores de YCF (AMTY), Sindicato de Obreros y Empleados Municipales (SOEM) y ATE 6 . Se trata de las Manzanas 14 y 24 que están en el Barrio YCF, las manzanas 43A y 42 (A y B) dentro del Barrio el Trencito.

\footnotetext{
${ }^{6}$ Aunque la Asociación de Trabajadores del Estado (ATE) no puede ser propietario por sus estatutos que no les permite recibir cosas del patrón, de ahí, que se forma la cooperativa al interior de YCF.
} 
Mz. 14. Barrio $\mathrm{YCF}^{7}$, ubicadas entre las calles Kirchner, Defensa, Zapiola y Estado de Israel, según el plano de Mensura Oficial de la Manzana, propiedad de YCF, del 1976, Plano No. 34D-1976. Está dividida por el pasaje Lago Burmeister, en dos secciones: A por calle Kirchner y B por calle Zapiola, de acuerdo a la Mensura oficial con subdivisión de la manzana, Plano. No. 91-P-1976, del año 1976. Se registran 20 viviendas en cada sección, siendo un total de 40. La mayoría mantienen su prototipo original de construcción (figura 10), aunque varias han sido modificadas.

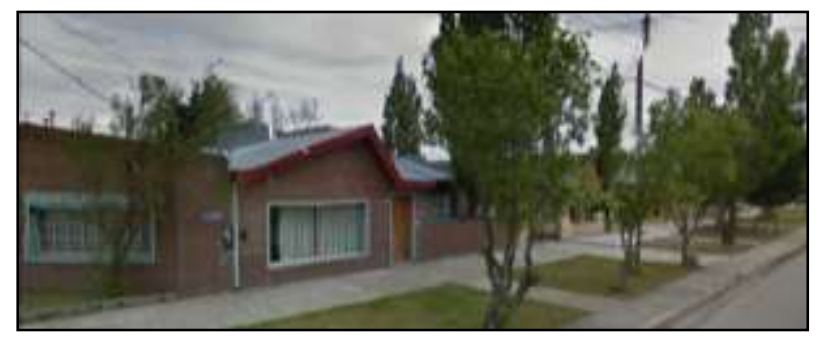

Figura 9. Casas de la manzana 231B. Foto: Google Earth.

Mz. 24. Situada sobre las calles de Alberdi, Baquiano Adolfo Parejas, Kirchner y Roundeau en el Barrio YCF. El primer registro catastral que se encuentra es la "Mensura de la Fracción I de YCF que comprende parte de las manzanas 1, 2, 3, 4, 14, 15, 16, 17, 23, 24, 31, 32 y 283 y tierras del sector nord-oeste" de propiedad de YCF, en el año 1971. Posteriormente aparece en la Mensura Oficial de la Manzana 24, propiedad de YCF, inscripto en el Registro de Subdivisiones el 30 de marzo de 1976, donde figura sin divisiones. El último registro es de la Mensura Oficial con subdivisión de la manzana 24, propiedad de YCF, aquí se aprecia la división por la calle Carbo Pereira, en dos secciones, A al norte y B al sur. Según el plano de mensura de subdivisión, cada sección tiene 20 lotes que se corresponden con las viviendas actuales. Las viviendas tienen la misma forma original (figura 10), que se puede aún distinguir más allá de las modificaciones.

Mz. 43 (mitad norte de la sección B). Ubicada entre las calles Avellaneda, Fitz Roy, Alberdi y Alberti. Actualmente está dividida, por la calle Saint Exupery, en dos secciones A y B. El registro catastral que se encontró sobre esta manzana es de una Mensura Administrativa con Subdivisión de las Manzanas No. 43, 44, 47 y 62, propiedad de YCF, con fecha de inscripción en el Registro de Subdivisiones del municipio del 3 de Junio de 1975. En la Sección B se encuentran casas construidas por YCF en la etapa 2, que ya se mencionó anteriormente. En la sección A, se aprecian viviendas que corresponden en su totalidad con el prototipo construido en las manzanas 14 y 24, por tanto, por analogía y por confirmación de esta deducción por parte de los referentes, se incluye en este trabajo. Esta manzana tiene la particularidad de tener galpones usurpados y convertidos en viviendas de lo que fue el matadero o frigorífico según los vecinos.

Mz. 42. Ubicada en las calles de Alberdi, Fitz Roy, Kirchner, Alberti. Esta manzana se divide en la sección A por la calle Alberti, y la sección B por la calle Fitz Roy. El primer plano de mensura sobre la manzana completa sin división data del año 1971, así aparece como antecedente en la Mensura Oficial de la Manzana 42 del año 1976, según el Plano No. 17-P1976. Posteriormente se realiza la división en la sección A y B en el año 1987, según consta en la Mensura Administrativa con subdivisión de la manzana 42, siendo propietario YCF.

\footnotetext{
${ }^{7}$ Nombre elegido por los vecinos en honor a la empresa. Es reconocido por la municipalidad en la Ordenanza No. 2949-HCD-01 de 2001. Sus límites son las calles Balbín, Chiclana y Avda. Almirante Brown.
} 


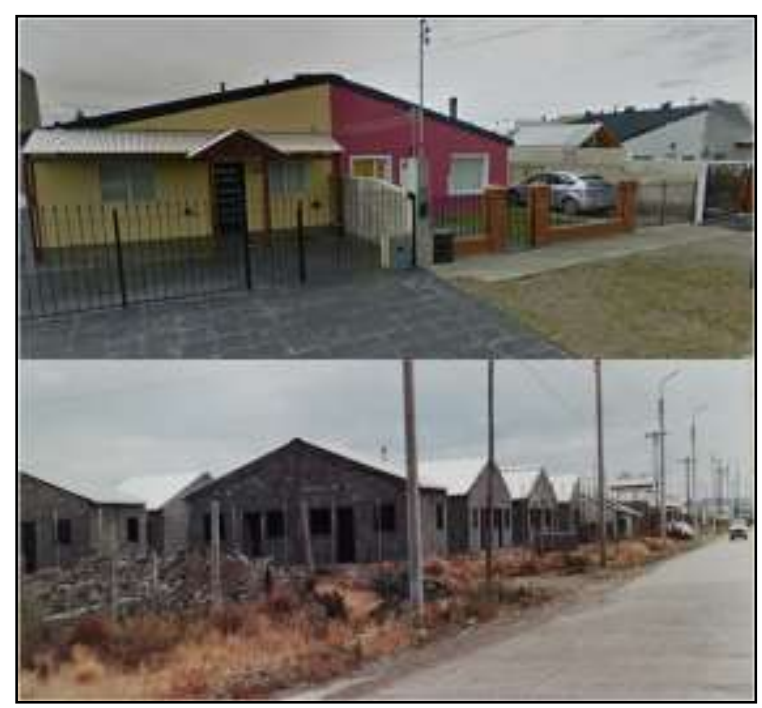

Figura10. Prototipo de casas del plan 200 viviendas. Foto: Google Earth (arriba) /González (abajo).

Sección A. Está compuesta con la Cooperativa de Consumo y Vivienda Patagónica Limitada, según una mensura del año 1994. No se obtuvo el dato de cuántos lotes le pertenecen. La sección se encuentra en las calles de Alberdi, Saint Exupery, Kirchner y Alberti. El prototipo es similar a las viviendas de las manzanas anteriormente descriptas. Consta de 20 lotes que corresponden a las 20 viviendas actuales, pocas presentan ampliaciones y solo una ha construido un primer piso.

En la sección B, ubicado en las calles de Alberdi, Fitz Roy, Kirchner y Saint Exupery. De los 20 lotes están construidas 15, y quedan 5 lotes vacíos por la calle Fitz Roy. Se observa mayor número de casas modificadas que la sección A.

En toda esta área, de acuerdo a la entrevista realizada, se sabe que se construyeron 125 viviendas, de las 200 posibles. Faltaban construir 75 casas más (por escasez de lotes), por tanto, los lotes se consiguieron en el Barrio Belgrano Viejo, a través del SOEM, pactando 50 viviendas para este organismo y 25 para $\mathrm{YCF}$, esto explica que la mensura esté a nombre de la Municipalidad de Río Gallegos y no de YCF (figura 11). En la distribución no se concentraron en una sola manzana a los trabajadores de YCF, sino que fue aleatorio. En este sector también se puede apreciar que las viviendas coinciden con el prototipo de las manzanas anteriores, pero no se cartografía porque se tendría que hacer a nivel lote y de esta manera se puede vulnerar el derecho a la privacidad del propietario. Pero es importante mencionarlo. Por último estas casas fueron entregadas en los primeros años de la década de los noventa.

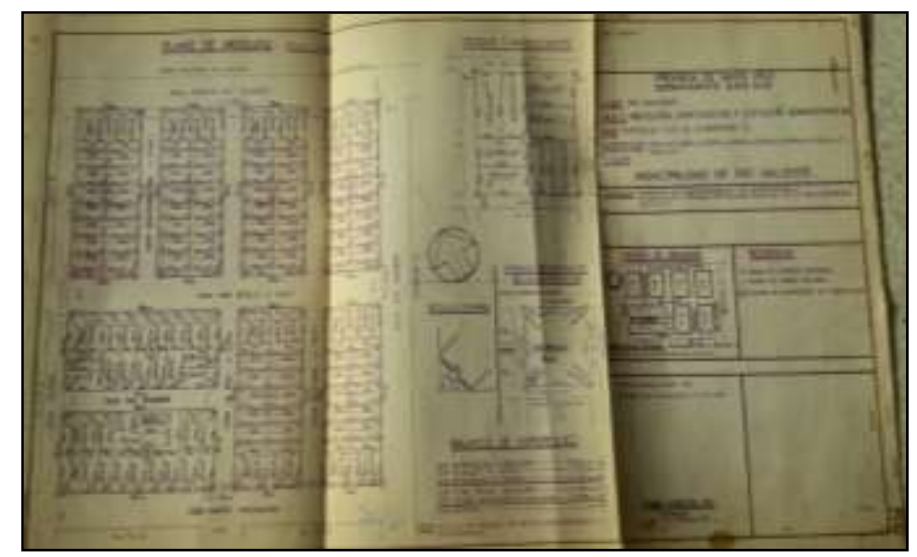

Figura11. Mensura de viviendas compartidas entre SOEM e YCF. Fuente: González. 


\section{Etapa 4.}

Corresponde a los años 2005 al 2013, son las más recientes. Forman parte de un plan nacional de 500 viviendas para trabajadores de YCRT, anunciado en el año 2005, a construirse en Río Turbio y Río Gallegos. Estas se construyeron a través del Instituto de Desarrollo Urbano y Vivienda (IDUV) entre los años 2011 y 2013. En Río Gallegos se componen de dos conjuntos habitacionales. El primero en la manzana 31 y el segundo en la manzana 48.

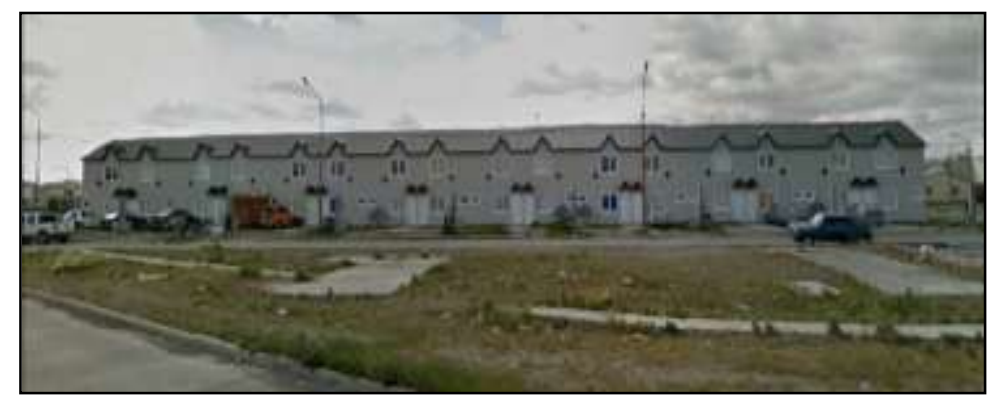

Figura12. Complejo de departamentos. Foto: Google Earth

Mz. 31. Sobre calles Alberdi, Alberti, Kirchner y Balbín. El registro catastral data del año 1971 con la "Mensura de la Fracción I de YCF que comprende parte de las manzanas 1, 2, 3, $4,14,15,16,17,23,24,31,32$ y 283 y tierras del sector nord-oeste" de propiedad de YCF. Un segundo registro se obtuvo de la Dirección de Informática del municipio donde aparece aún como único propietario de todo el predio YCF, bajo el Instrumento Legal T60F15F1381. Aquí se construyeron 36 departamentos, de dos pisos, divididos en dos conjuntos paralelos a la calle Alberti y Haití, uno con 20 departamentos y otro con 16, respectivamente. Son de color gris claro con techo negro. De a poco se comienza a ver ciertas modificaciones en cuanto a la colocación de medianeras que dividen las entradas de las viviendas (figura12).

Mz. 48. Está sobre las calles Avellaneda, Catamarca, Alberdi y Fitz Roy. Se obtuvo registro de la Dirección de Informática del municipio donde figura YCF como único propietario de toda la manzana, bajo el Instrumento Legal T59F101F989. Se trata de una manzana dividida, por un pasaje sin nombre, en dos secciones, una al norte y otra al sur. Aquí se construyeron 36 casas, abarcando la totalidad de la manzana. Se distinguen por ser en color gris claro con el techo negro de dos aguas (figura 13). Aún sin árboles, recientemente habitadas. Según el sitio de noticias online PatagoniaNexo, estas viviendas fueron entregadas a finales del año 2013.

Se conoció que los fondos para construir estas viviendas provenían del Ministerio de Planificación quien le otorgaba el dinero a YCRT y éste al IDUV, sin embargo por problemas en los primeros dos adelantos de obra que se pagaron, se decidió que YCRT transfiriera directamente el dinero a las empresas particulares contratadas para la ejecución de la obra.

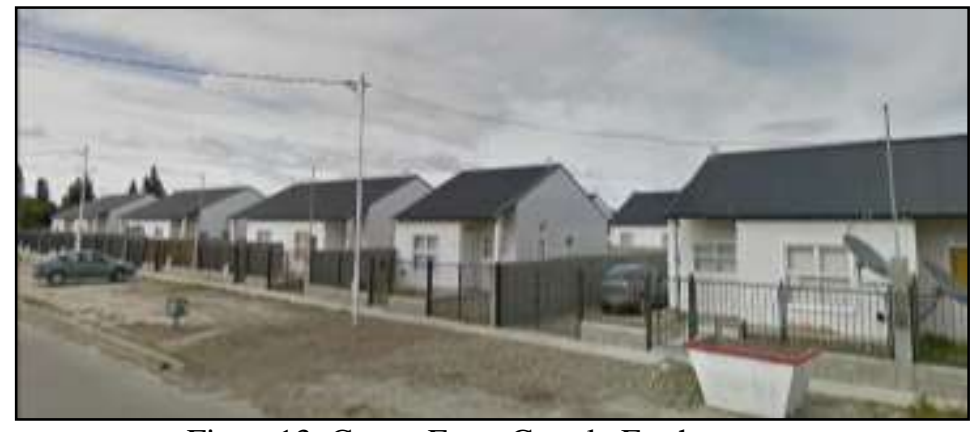

Figura13. Casas. Foto: Google Earth

Otros datos importantes: Con respecto a quiénes fueron los beneficiarios de las casas y cómo fueron los planes de entrega, los referentes de YCRT, dijeron que para la primera etapa solo 
recuerdan que debían ser empleados de la empresa y formar parte de la planta permanente. Sin embargo para las casas entregadas en la década de 1970, segunda etapa, el beneficiario debía ser de planta permanente en la empresa, con al menos 5 años de antigüedad. Fue pensado para familias, por lo tanto los empleados solteros no pudieron acceder a alguna vivienda. Sobre el pago, en esta etapa y en las anteriores, se descontaba de los haberes. Hoy un amplio porcentaje de los beneficiarios ya tiene título de propiedad, incluso algunas ya han cambiado de propietario. Para la tercera etapa se debían reunir casi los mismos requisitos anteriores. Y para el cuarto plan de viviendas, sí se tomó en cuenta al personal soltero, actualmente ninguno tiene título de propiedad, ya que están en comodato y están determinando el mecanismo de pago, que posiblemente sea a través de un crédito bancario.

Con respecto al predio del puerto y los alrededores se pueden apreciar los vestigios de la infraestructura en la época del trencito, se sabe que si bien pertenecen a YCF, han estado bajo el resguardo de lo ONABE, (Organismo Nacional de Administración de Bienes del Estado), hoy AABE (Agencia de Administración de Bienes del Estado). La AABE junto a YCRT han creado un plan de limpieza de todos esos predios con el fin de mejorar la imagen y poner en valor esa parte de la costanera.

Por último a modo de síntesis de lo anteriormente descrito, se presenta la cartografía, donde se identifican verdaderos conglomerados para la aplicación de encuestas en posteriores estudios.

Figura 14. "Manzanas surgidas a partir del carbón".

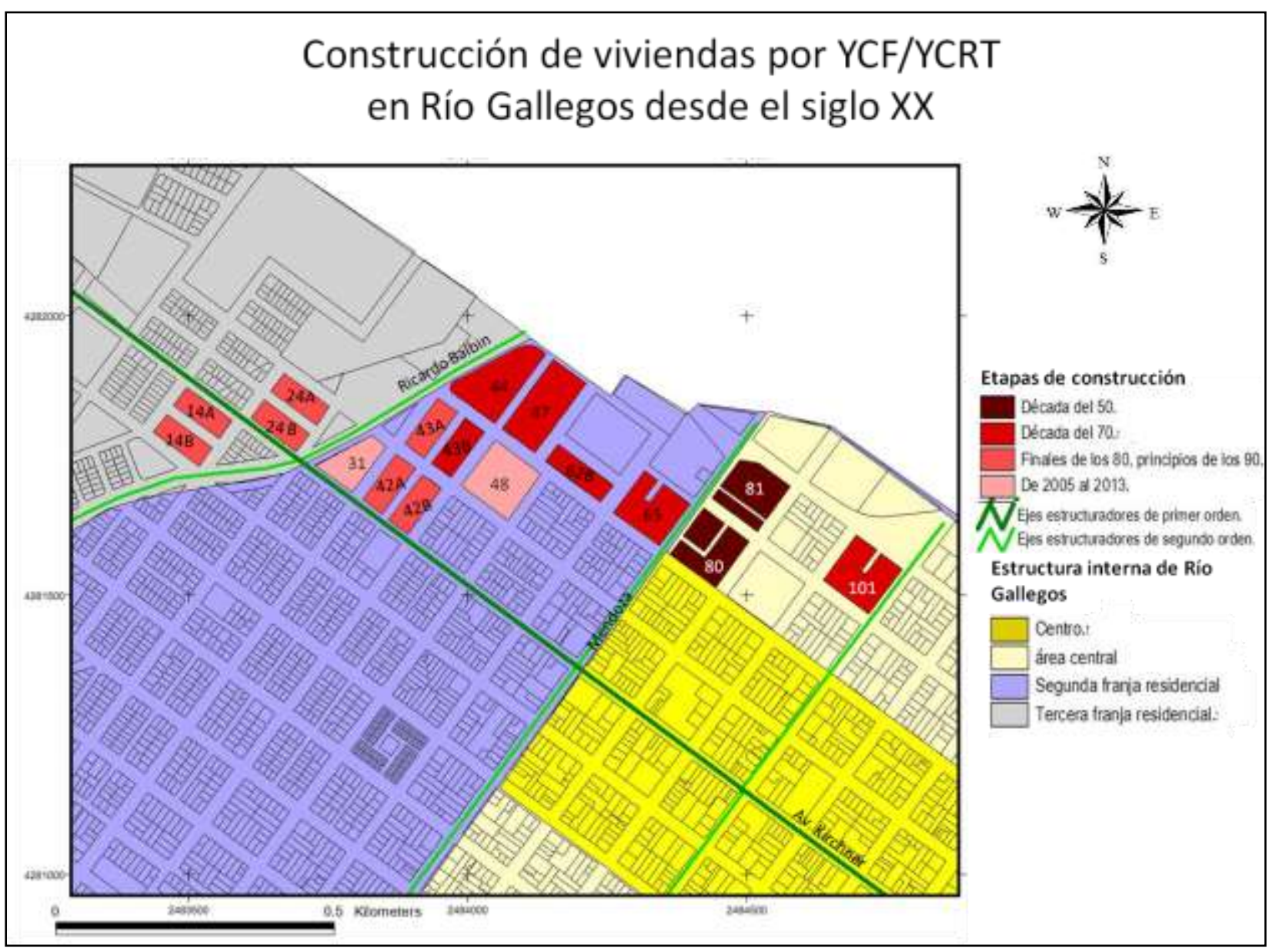

Fuente: Laboratorio de Cartografía UNPA. Elaboración: Karla L. Aceves Padilla. 


\subsection{Discusión}

Con relación al concepto de Barrio:

Uno de los objetivos propuestos al inicio de la investigación era el identificar barrios surgidos a consecuencia de la actividad extractiva del carbón. De acuerdo con esto y los resultados se puede afirmar que hubo dos barrios cuyo origen tiene que ver directamente con esta actividad. Uno es el barrio el Trencito, y otro es el barrio YCF. Independientemente de que el resto de las manzanas que conforman el barrio, no se hayan podido rastrear como parte de los planes de viviendas impulsadas por la empresa YCF/YCRT, no se puede negar que su origen tuvo que ver de alguna u otra manera a las actividades que ahí se venían realizando a consecuencia de la dinámica generada por el puerto y el ramal ferroviario, es decir, por la "dinámica del carbón". Recorrer las calles, las manzanas, el barrio, es encontrarse permanentemente con aquélla época a través de las casas de durmientes, del museo ferroviario de las instalaciones de YCRT, del Club Ferro (figura15), de los vecinos que se sienten orgullosos (más allá de que varios de ellos piensen que ya se perdió mucha identidad), y sobre todo porque desde cualquier punto se observa el puerto, que da cierta nostalgia. Es aquí donde radica la esencia de un barrio y es aquí donde Zamorano recobra importancia, pues afirma que el barrio es un conjunto que constituye un medio de vida y de actividades dotado de individualidad, que implica una vivencia especial para los ciudadanos, y esta vivencia especial es lo que se logró percibir en los vecinos de este lugar.

Por otro lado, también Zamorano afirma que cuando una superficie es circunscrita como un espacio de administrativo, de gestión, suele pasar que no existe una coincidencia con el barrio en cuanto a lo que los vecinos experimentan, esta situación es muy notable en las áreas estudiadas. Por un lado, se nota un conflicto entre el nombre del barrio El Trencito y la delimitación de sus calles, reconocidas ya por la municipalidad.

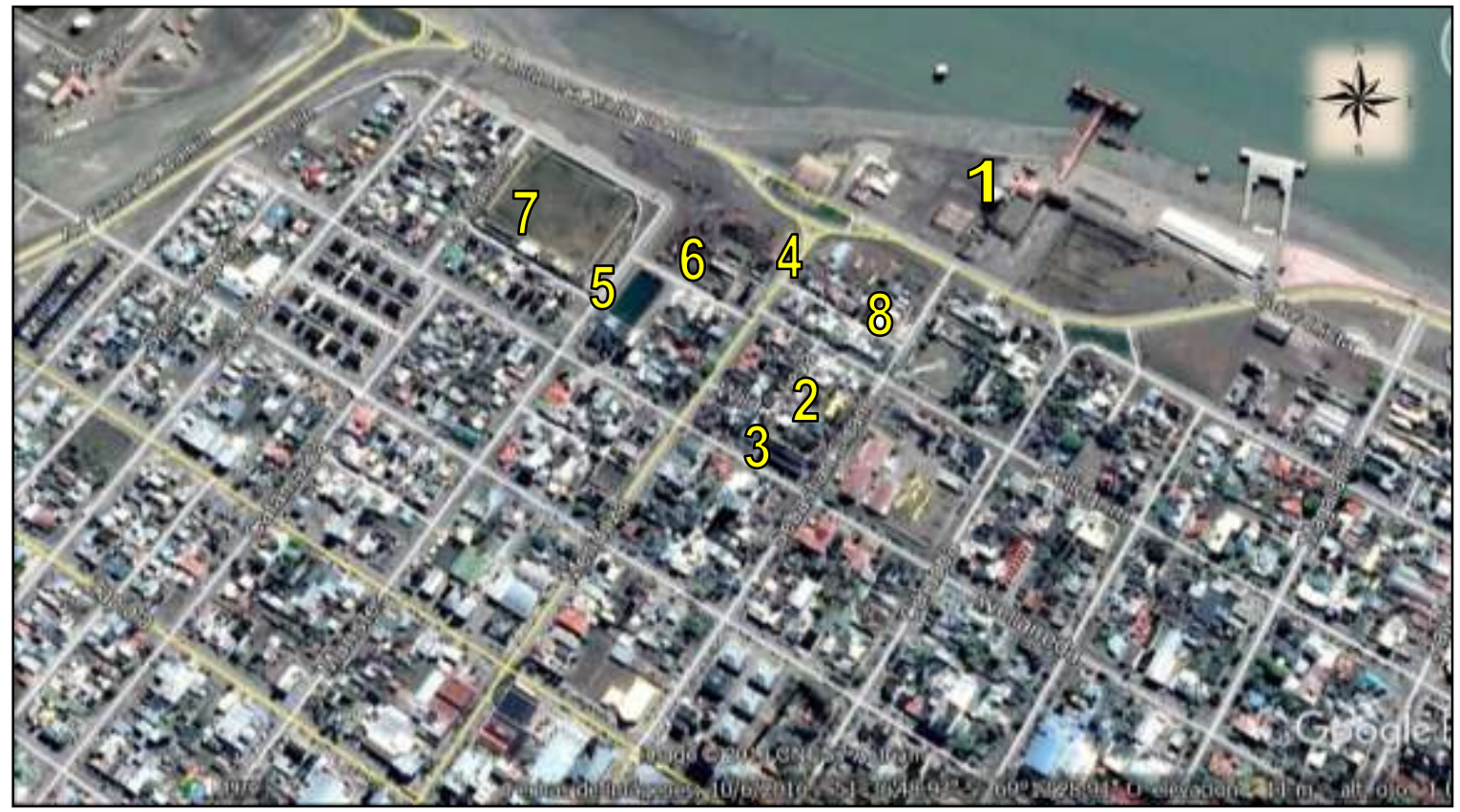

Figura 15. Ubicación de elementos que dan identidad al barrio.

1) Instalaciones Portuarias

2) Oficinas de YCRT

3) Ministerio de la Producción

4) Centro de Jubilados y Pensionados de YCF
5) Cancha Fútbol de YCRT

6) Museo Ferroviario

7) Club Deportivo Ferro

8) Cap. Virgen Niña 
Anteriormente toda esa área era conocida como Barrio el Puerto, y después a través de las juntas vecinales se decidió cambiar al nombre de El Trencito, hoy, administrativamente así figura. Sin embargo algunos vecinos ignoran este cambio y lo siguen llamando el Puerto, de hecho manifestaron que les gustaría que se siguiera llamando igual. Por otro lado, la municipalidad no toma en cuenta cierta identidad del barrio, pareciera ser que el criterio que rige a la hora de demarcar un barrio, sería la practicidad y no la historia e identidad.

Cáceres (2000) afirma que el paisaje urbano es el resultado de componentes formales relacionados con el marco natural, y que el plano es el resultado de la combinación sobre el espacio de la ciudad a través del tiempo, de superficies libres (calles, parques, jardines, plazas, etc.) y de superficies construidas. Los ejes de fijación naturales (río, bahía, curva de nivel) y humanos (vías férreas, rutas) influyen en la orientación del plano. En este sentido la costanera ha marcado un límite de crecimiento del barrio hacia el sector norte. Y las vías férreas han influido en el tamaño y forma de las manzanas, por ende las casas que se encuentran alrededor de la calle Balbín, tal es el caso del conjunto de 36 departamentos construidos en la última etapa, que rompen la forma de edificación de las manzanas aledañas.

Con relación a los usos de suelo, predomina el residencial, y en menor grado el administrativo, por último el comercial. Según el marco teórico, el barrio residencial es el que ocupa mayor superficie en las ciudades, aunque también influye que la investigación se orientó a manzanas residenciales.

Por otro lado, y aludiendo a la aportación del Ing. Williams sobre los solares a, b, c, d, se pudo observar, en algunos de los planos de mensura consultados, esta división.

Según la distribución de las manzanas encontradas, se observa que la calle Av. Balbin y las manzanas baldías que hay en ella provoca un quiebre importante que podría afectar a una futura propuesta que incluya a todas ellas en una nueva delimitación como parte de un mismo barrio, con un nombre que las identifique y que resalte su identidad, sin que se sienta la cierta rivalidad que hoy se percibe entre los que viven en el Trencito y los que viven en el Puerto, (rivalidad que esconde un orgullo muy grande por pertenecer a uno y a otro). Se podría realizar a través de la reactivación de las juntas vecinales y mediante una elección que incluya a todos los vecinos involucrados, (como ya lo hizo el barrio YCF), y una vez, saltando esa barrera, se podrían agrupar y darle una identidad propia que rescate su historia y sus valores. Por último, el mapa de ubicación de las manzanas, es la síntesis que sienta un antecedente importante. Se espera que ayude a la gestión de las mismas y a futuros investigadores de estos temas. El hecho de contar con los documentos catastrales, lo convierten en una fuente de información que ayudará a reducir los tiempos en las investigaciones futuras.

\section{CONCLUSIONES}

La hipótesis del trabajo fue: "desde el siglo XX, han surgido barrios vinculados a la actividad carbonífera, destinados a trabajadores de YCF-YCRT. Actualmente hay un sector que no ha sido comprendido como parte del barrio El Trencito". Efectivamente hay un sector no comprendido en el Barrio el Trencito y son las manzanas 14 y 24 ubicados en el barrio YCF, descubriendo, además, que en el Barrio Belgrano Viejo se destinaron 25 viviendas a empleados de YCF, sin embargo no se concentraron en un solo sector, sino que están esparcidos entre otras viviendas del SOEM. Así mismo se mencionan también las viviendas del Barrio Jardín, pero por la cantidad (la manzana está compartida entre YPF, YCF y la Caja 
de Previsión Social de la Provincia) no llegan a constituir identidad propia y fueron absorbidas por la identidad de ypefiano, ya que se encuentran en el Barrio YPF Jerárquico ${ }^{8}$. Dado los tres criterios establecidos para elegir las manzanas a cartografiar, se dejaron de lado muchas manzanas que tienen amplias posibilidades de encontrar un origen en la actividad del carbón. ¿Por qué se llega a esta conclusión?, porque inicialmente los terrenos de YCF abarcaban un área muy amplia (como se pudo observar en el plano del puerto), por lo tanto abarcan muchas manzanas más, y hoy la mayoría de esas manzanas están urbanizadas, entonces ¿cómo fue que esas manzanas se urbanizaron?, en algún momento YCF se debió haber desprendió de ellas. ¿Cómo, en qué momento se realizó y bajo qué circunstancias? Este sería un tema para un segundo trabajo de investigación a modo de ampliación.

\section{RECOMENDACIONES}

Los resultados de esta investigación se recomienda ser tratados con toda la responsabilidad profesional necesaria, esto implica, no alterarlos, ni reproducirlos sin citar a las autoras.

Tómese en cuenta que hay manzanas que si bien figuran cartografiadas, no se contó con el plano de mensura, pero sí, con el número de instrumento legal encontrados en los archivos de informática del municipio. Cabe aclarar que la fecha de un plano de mensura no necesariamente indica los años de construcción de las viviendas, por ejemplo, la manzana de las casas de durmientes datan de los años 50, sin embargo, el plano de mensura es del año 72 y 74.

Por otra parte, se recomienda a las dependencias gubernamentales tener un protocolo de atención al alumno o docente investigador. Deben ser conscientes que son una fuente de información directa y la atención no puede estar supeditada a la cantidad de trabajo que tenga la dependencia. El investigador debe ser atendido en tiempo y forma, pues de alguna manera su trabajo puede ser de gran utilidad para la sociedad y los entes estatales.

\section{AGRADECIMIENTOS}

A Esteban Gesto, Director de Informática en la Municipalidad de Río Gallegos

A Luis Peralta, de YCRT, por dar las primeras pistas.

A Luis Héctor González, por su valioso aporte.

A la Dirección de Catastro Municipal de Río Gallegos.

Al Archivo Municipal de Río Gallegos.

Al personal de YCRT.

A la Lic. Alicia P. Cáceres, por su paciencia.

A la Prof. Mónica Norambuena por su orientación y disponibilidad.

Al Ing. Gerardo Riesco, mi esposo, por su asesoría en los planos de mensura.

A mi mamá, mi papá y mi hermana, por estar.

\section{REFERENCIAS}

ACEVES y CÁCERES 2015. Identificación geográfica de Barrios residenciales surgidos a partir de la actividad hidrocarburífera, en Río Gallegos durante el siglo XX". ICT UNPA, ISSN-e 1852-4516, Vol. 7, Nº. 2, 2015, págs. 23-57

\footnotetext{
${ }^{8}$ Bajo este nombre se identificaron a las casas ubicadas en el Barrio Jardín, que eran utilizadas por el personal jerárquico de YPF en el ICT "Identificación geográfica de barrios residenciales surgidos a partir de la actividad hidrocarburífera en Río Gallegos durante siglo XX” realizada por la autora de este trabajo. Las manzanas están entre Mayer, Villarino, Mitre y Mascarelo.
} 
AGUIRRE 2000. Río Gallegos, capital de la provincia. El Gran Libro de Santa Cruz. Alfa Centro Literario y Editorial Oriente. España. ISBN O.CL: 987-99023-1-9.

BARBERÍA 1985. Distribución de la Tierra pública en Río Gallegos. El centenario de Río Gallegos 1985-1995. Argentina. Municipalidad de Río Gallegos. ISBN 950-43-07272.

BONA 2000. Actividades económicas en Santa Cruz -1940-1990. El Gran Libro de Santa Cruz. España. ISBN O.CL 987-99023-1-9.

CÁCERES 2000. Estructura y paisajes urbanos de Santa Cruz. El Gran Libro de Santa Cruz. Alfa Centro Literario y Editorial Oriente. España. ISBN O.CL: 987-99023-1-9.

CÁCERES, et. al. 2012. La ciudad, espacio de significados visible en el paisaje urbano. El caso de la costanera de Río Gallegos. VI Jornadas de Comunicación Social. Universidad Nacional de la Patagonia Austral. CD-ROM. ISBN 978-987-1242-70-2.

CÁCERES, et. al. 2013. Consolidación de la Tercera Franja Residencial de Río Gallegos, Patagonia Austral. Contribuciones científicas GAEA. Vol 25. Pags. 47-59.

CÁCERES 2013. Crecimiento urbano de la ciudad de Río Gallegos. Capital de la Provincia de Santa Cruz, Patagonia Austral Argentina (1885-2010). Revista Científica de Geografia. Geousal. Año VIII No 14.

CÁCERES y GARCÍA 2004. Diferenciación interna del espacio geográfico de Río Gallegos: estudio preliminar. Párrafos Gráficos. Año III. N³ IGEOPAT.

Carpeta de calles. Legislación vigente. Listado. Honorable Concejo Deliberante.

Carpeta de Barrio, Complejos y Conjuntos habitacionales. Legislación vigente. Ordenanzas y Decretos. Honorable Concejo Deliberante.

CUFRÉ y ZONARO. 1985. Análisis de la tipología de vivienda patagónica en Río Gallegos. El centenario de Río Gallegos 1985-1995. Argentina. Publica la Municipalidad de Río Gallegos. ISBN 950-43-0727-2.

FERRO 2006. Las casas de durmientes de Yacimientos Carboníferos Fiscales.

MENCO, 2016. Delimitar los barrios construidos por el Estado Nacional, Provincial y Municipal antes de 1990 a través de un SIG. Informe Científico Técnico UNPA ISSN: $1852-4516$

MUÑOZ SESNIC, NORAMBUENA y CÁCERES 2011. Las transformaciones en el paisaje urbano de Río Turbio en el siglo XX. Patagonia Andina de la Argentina Austral. En Actas Científicas CNG $72^{\circ}$ Semanas de Geografía.

PERAZZO, M. 1985. Temas históricos de Río Gallegos. El centenario de Río Gallegos 19851995. Argentina. Publica la Municipalidad de Río Gallegos. ISBN 950-43-0727-2.

ZAMORANO 1992. Geografía Urbana: formas, funciones y dinámica de las ciudades. Buenos Aires. Colección Geográfica. Ed. Ceyne.

\section{Direcciones Web}

- Sobre información de YCRT: www.ycrt.gob.ar

- Para el concepto de "durmientes": www.listadepalabras.es/palabra_significado.php?woordid=DURMIENTE

- Para la entrega de casas última etapa: www.patagonianexo.com.ar/v2/se-entregaron-viviendas-de-ycrt-en-rio-gallegos/ 